\title{
Chandra High Energy Transmission Gratings Spectra of V3890 Sgr
}

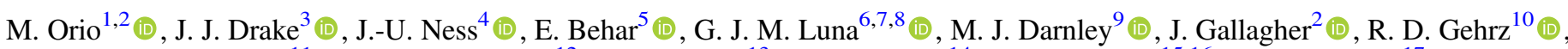

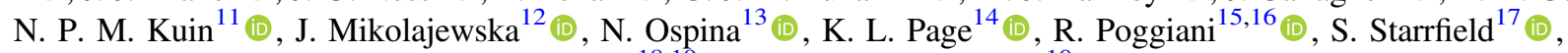 \\ R. Williams ${ }^{18,19}$ (D), and C. E. Woodward ${ }^{10}$ (iD \\ ${ }^{1}$ INAF-Osservatorio di Padova, Vicolo Osservatorio 5, I-35122 Padova, Italy; orio@astro.wisc.edu \\ ${ }^{2}$ Department of Astronomy, University of Wisconsin, 475 N. Charter Street, Madison, WI 53704, USA \\ ${ }^{3}$ Harvard-Smithsonian Center for Astrophysics, 60 Garden Street, Cambridge, MA 02138, USA \\ ${ }^{4}$ XMM-Newton Science Operations Center, European Space Astronomy Center, Camino Bajo del Castillo s/n, Urb. Villafranca del Castillo, E-28692 Villanueva de \\ la Cañada, Madrid, Spain \\ ${ }^{5}$ Department of Physics, Technion, Haifa, Israel \\ ${ }^{6}$ CONICET-Universidad de Buenos Aires, Instituto de Astronomia y Fisica del Espacio (IAFE), Av. Int. Güiraldes 2620, C1428ZAA, Buenos Aires, Argentina \\ ${ }^{7}$ Universidad de Buenos Aires, Facultad de Ciencias Exactas y Naturales, Buenos Aires, Argentina \\ ${ }^{8}$ Universidad Nacional de Hurlingham, Tte. Origone 151, Hurlingham, Buenos Aires, Argentina \\ ${ }^{9}$ Astrophysics Research Institute, Liverpool John Moores University, Liverpool, L3 5RF, UK \\ ${ }^{10}$ Minnesota Institute for Astrophysics, University of Minnesota, 116 Church Street S.E, Minneapolis, MN 55455, USA \\ ${ }^{11}$ University College London, Mullard Space Science Laboratory, Holmbury St. Mary, Dorking RH5 6NT, UK \\ ${ }^{12}$ Nicolaus Copernicus Astronomical Center, Polish Academy of Sciences, Bartycka 18, PL-00-716 Warszawa, Poland \\ ${ }^{13}$ Department of Physics and Astronomy, University of Padua, vicolo Osservatorio, 3, I-35122 Padova, Italy \\ ${ }^{14}$ School of Physics and Astronomy, University of Leicester, University Road, Leicester LE1 7RH, UK \\ ${ }^{15}$ Dipartimento di Fisica, Università di Pisa, I-56127 Pisa, Italy \\ ${ }^{16}$ INFN, Sezione di Pisa, I-56127 Pisa, Italy \\ ${ }^{17}$ Department of Earth and Space Exploration, Arizona State University, P.O. Box 871404, Tempe, AZ 85287-1404, USA \\ ${ }^{18}$ Department of Astronomy \& Astrophysics, University of California, Santa Cruz, 1156 High Street, Santa Cruz, CA 95064, USA \\ ${ }^{19}$ Space Telescope Science Institute, 3700 San Martin Drive, Baltimore, MD 21218, USA \\ Received 2020 February 14; revised 2020 April 20; accepted 2020 April 21; published 2020 May 28
}

\begin{abstract}
The recurrent nova (RN) V3890 Sgr was observed during the seventh day after the onset of its most recent outburst, with the Chandra ACIS-S camera and High Energy Transmission Gratings. A rich emission line spectrum was detected, due to transitions of $\mathrm{Fe}-\mathrm{L}$ and $\mathrm{K}$-shell ions ranging from neon to iron. The measured absorbed flux is $\approx 10^{-10} \mathrm{erg} \mathrm{cm}^{-2} \mathrm{~s}^{-1}$ in the $1.4-15 \AA$ range $(0.77-8.86 \mathrm{keV})$. The line profiles are asymmetric, blueshifted, and skewed toward the blue side, as if the ejecta moving toward us are less absorbed than the receding ejecta. The full width at half-maximum of most emission lines is $1000-1200 \mathrm{~km} \mathrm{~s}^{-1}$, with some extended blue wings. The spectrum is thermal and consistent with a plasma in collisional ionization equilibrium with column density $1.3 \times 10^{22} \mathrm{~cm}^{-2}$ and at least two components at temperatures of about 1 and $4 \mathrm{keV}$, possibly a forward and a reverse shock, or regions with differently mixed ejecta and a red giant wind. The spectrum is remarkably similar to the symbiotic RNe V745 Sco and RS Oph, but we cannot distinguish whether the shocks occurred at a distance of a few au from the red giant, or near the giant's photosphere, in a high-density medium containing only a low mass. The ratios of the flux in lines of aluminum, magnesium, and neon relative to the flux in lines of silicon and iron probably indicate a carbon-oxygen white dwarf.
\end{abstract}

Unified Astronomy Thesaurus concepts: Novae (1127); Recurrent novae (1366); Cataclysmic variable stars (203); X-ray astronomy (1810); Atomic spectroscopy (2099); High resolution spectroscopy (2096)

\section{Introduction}

Recurrent novae (RNe), like all novae, are interacting binaries with a white dwarf (WD) that accretes matter from its companion and undergoes a thermonuclear runaway (TNR). They are called "recurrent" because they have been observed in outburst more than once, although all novae are thought to have repeated outbursts on different, secular timescales (e.g., Prialnik 1986). Only a small number of RNe are known, 10 in the Galaxy (see the review by Orio 2015), 3 in the Large Magellanic Cloud (Bode et al. 2016; Mróz et al. 2016) with a recent additional candidate (Iłkiewicz et al. 2019), and 12 in M31 with 4 additional candidates (Shafter et al. 2015). Of the 10 known Galactic RNe, 4 are "symbiotic novae," relatively rare novae in long orbital period systems with a red giant companion.

V3890 Sgr is one of the symbiotic RNe, and it was observed in outburst for the third time on 2019 August 27.87 (it was reported by A. Pereira, see Strader et al. 2019). Previous outbursts occurred in 1962 and 1990, spaced $28 \mathrm{yr}$ apart. The last quiescent optical photometric observation was made less than a day before the outburst detection (Strader et al. 2019), so the outburst time is fairly well constrained. Table 1 summarizes the known physical parameters for V3890 Sgr. The current outburst of V3890 Sgr may have been faster, with shorter $t_{2}$ and $t_{3}$ (times to drop 2 and and 3 mag from maximum optical luminosity, respectively) than the previous one, according to the AAVSO publicly available light curve. However, it is possible that the initial phases in the previous eruptions of 1962 and 1990 were missed, so that $t_{2}$ and $t_{3}$ may not have been well constrained. The time to decay by 6 magnitudes was about the same in each outburst (Strope et al. 2010, and references therein).

For a useful comparison, Table 1 also includes the known parameters for the other three Galactic symbiotic $\mathrm{RNe}$, which have been observed and scrutinized in the past much more extensively than V3890 Sgr, especially RS Oph. We note that 
Table 1

Physical Parameters of the Known Symbiotic Recurrent Novae

\begin{tabular}{|c|c|c|c|c|c|c|c|c|c|c|c|c|}
\hline Name & $\begin{array}{c}m(\mathrm{WD}) \\
\left(M_{\odot}\right)\end{array}$ & $\begin{array}{c}m \text { (giant) } \\
\left(M_{\odot}\right)\end{array}$ & $\begin{array}{l}\text { Spectral } \\
\text { Type }\end{array}$ & $\begin{array}{c}d \\
(\mathrm{kpc})\end{array}$ & $\begin{array}{c}P_{\text {orb }} \\
\text { (days) }\end{array}$ & $\begin{array}{c}t(\mathrm{rec}) \\
(\mathrm{yr})\end{array}$ & $V_{\max }$ & $\begin{array}{c}v(\mathrm{ej}) \\
\left(\mathrm{km} \mathrm{s}^{-1}\right)\end{array}$ & $\begin{array}{c}t_{2} \\
\text { (days) }\end{array}$ & $\begin{array}{c}t_{3} \\
\text { (days) }\end{array}$ & $\begin{array}{c}t_{6} \\
\text { (days) }\end{array}$ & $\begin{array}{c}\text { Times(SSS) } \\
\text { (days) }\end{array}$ \\
\hline V3890 Sgr & & & M5 III $(1,2)$ & $\begin{array}{l}4.36_{-1.31}^{+2.64} \\
\geqslant 4.5(5)\end{array}$ & (see 3) & 28 & 7 & $\geqslant 4200(4)$ & $\begin{array}{l}6 \\
2\end{array}$ & $\begin{array}{c}14 \\
5\end{array}$ & $\begin{array}{l}28 \\
27\end{array}$ & $8-20-26^{a}$ \\
\hline RS Oph & $1.2-1.4(6)$ & $0.68-0.80(6)$ & M0-2 III (6) & $\begin{array}{c}2.29_{-0.27}^{+0.28} \\
1.6(9)\end{array}$ & $453.6(6)$ & $\approx 10^{\mathrm{b}}$ & 4.8 & $4200(7)$ & 7 & 14 & 88 & $26-58-86(8)$ \\
\hline V745 Sco & $>1.3(10)$ & & M6 \pm 2 III (1) & $\begin{array}{c}7.28_{-2.92}^{+4.52} \\
7.8 \pm 1.8(10)\end{array}$ & & $26 ?$ & $9.6(1)$ & $\geqslant 4000(11)$ & 2 & 4 & & 4-6-7 (12) \\
\hline $\mathrm{T} \mathrm{CrB}$ & $\begin{aligned} 1.2 & \pm 0.2(13) \\
1.37 & \pm 0.13(14)\end{aligned}$ & $\begin{array}{c}0.7(13) \\
1.12 \pm 0.23(16)\end{array}$ & M4 III (14) & $0.822_{-0.076}^{+0.034}$ & $227.67(15)$ & 80 & 2 & & 4 & 6 & 15 & \\
\hline
\end{tabular}

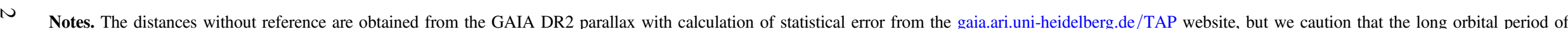

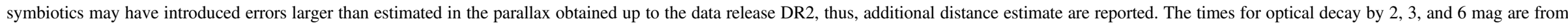

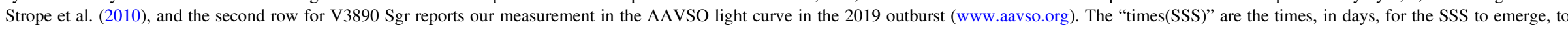
remain at maximum luminosity, and to fade beyond detection, respectively.

${ }^{a}$ K.L. Page et al. (2020, in preparation).

${ }^{\mathrm{b}}$ However, the last outburst of RS Oph occurred only after $21 \mathrm{yr}$ and no new outburst has been recorded in the last $13.5 \mathrm{yr}$.

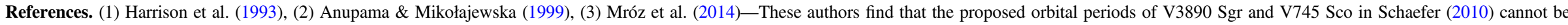

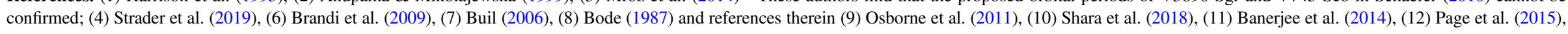
(13) Belczynski \& Mikolajewska (1998), (14) (Mürset \& Schmid 1999), (15) Lines et al. (1988), (16) Stanishev et al. (2004). 
all four objects have an $\mathrm{M}$ spectral type companion and are fast novae with short $t_{2}$ and $t_{3}$. We also know that the orbital periods exceed one year in RS Oph and V745 Sco. Symbiotic RNe are somewhat outliers among novae in several ways. The WD mass, $m(\mathrm{WD})$, has been estimated to be very high for RS Oph, V745 Sco, and T CrB. As for most RNe, the maximum absolute optical magnitude cannot be empirically related to the time for a decay of 2 or 3 mag via the maximum-magnituderate-of-decay relationship (MMRD; see della Valle \& Livio 1995). The ejected material in V3890 Sgr, as well in the other symbiotic RNe in Table 1 , had high velocity, reaching at least $4000 \mathrm{~km} \mathrm{~s}^{-1}$. This fact is not yet explained by the models of the TNR, which indicate slower ejecta than observed for several RNe, both symbiotic and otherwise (Yaron et al. 2005). The short interoutburst times imply high accretion rates, $\dot{m} \geqslant 10^{-8} M_{\odot} \mathrm{yr}^{-1}$, necessary to build enough pressure at the base of the envelope and start the TNR again every few years or tens of years (e.g., Yaron et al. 2005). RS Oph and T CrB are thought to be disk accretors, like generally many other symbiotics, although this does not imply that all symbiotics with a disk fill their Roche lobe. In fact, most symbiotics do not fill it, although several of them, never observed in nova outburst, show signatures of semi-steady nuclear burning (see Orio et al. 2007; Mikołajewska 2012; Orio 2013), requiring even higher $\dot{m}$ than in RNe. Such high $\dot{m}$ is not reached with Bondi-Hoyle accretion (Bondi \& Hoyle 1944), yet in a few cases, the system parameters are known with sufficient precision to rule out Roche lobe-filling. Often, ellipsoidal variations are observed, implying that some symbiotics are tidally distorted, and for this reason, some material indeed flows through the inner Lagrangian point L1 (see Mikołajewska 2012, and references therein). In other symbiotics, including the well-studied RS Oph, no tidal variations are observed, but there are signatures of a disk. Mohamed et al. (2013) suggested that the stellar wind of the RS Oph giant flows through the L1 point because it is gravitationally focused and flows in a spiral shock on the equatorial plane.

Because of the circumstellar red giant wind, the nova ejecta in symbiotic novae impact colder and slowly moving material (with a velocity of about only $10 \mathrm{~km} \mathrm{~s}^{-1}$ ), causing strong shocks that are observed in X-rays. Like other symbiotic novae, symbiotic RNe should also emit significant gamma-ray flux, as observed in the first nova detected with Fermi-LAT, V407 Cyg, which is also a symbiotic (Abdo et al. 2010). However, V745 Sco, the first symbiotic RN in outburst in the Fermi era, was only marginally detected with Fermi (see Cheung et al. 2014; Orio et al. 2015, and references and discussion therein).

The new outburst of V3890 Sgr has been monitored at all wavelengths with many different instruments, also because RNe with high-mass WDs (see Table 1) are interesting as possible progenitors of SNe Ia while they are still "single degenerates" (e.g., Starrfield et al. 2019). Early optical and near-infrared spectra have been described, among others, by Strader et al. (2019), Evans et al. (2019), Rudy et al. (2019), Munari \& Walter (2019a, 2019b), Pavana et al. (2019a), and Woodward et al. (2019). An ultraviolet spectrum was obtained by Kuin et al. (2019), and Swift also provided the UV light curve. In Figure 1 we present the "early" Swift X-Ray Telescope (XRT) light curve. Hard X-rays were immediately detected with the Swift XRT (Sokolovsky et al. 2019), and the nova was a gamma-ray source (Buson et al. 2019), as expected. Already on the second day after the eruption, the Swift X-ray

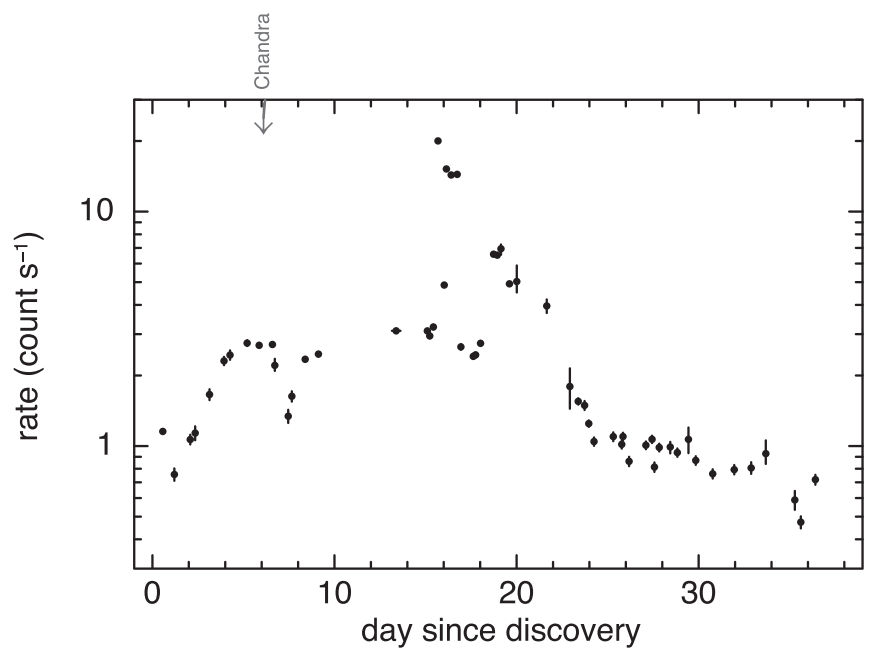

Figure 1. The light curve of V3890 Sgr measured with the Swift XRT in the $0.5-10 \mathrm{keV}$ range. The date of the Chandra observation is indicated by the arrow. An XRT light curve measured over a long period is included in Page et al. (2020, in preparation).

spectrum showed a hard X-ray source that could be fitted as a thermal plasma with $k T=7.9_{-1.6}^{+2.3} \mathrm{keV}$ (Sokolovsky et al. 2019). No connection can be made between the measured $\mathrm{X}$-ray emission and the gamma-ray emission because some nonthermal X-ray flux is also expected when gamma-rays are observed (Vurm \& Metzger 2018), and only a thermal spectrum was observed with Swift by Sokolovsky et al. (2019). A radio detection was announced by Pavana et al. (2019b) with likely thermal emission, but nonthermal synchrotron emission was later also detected (Pavana et al. 2019b; Polisensky et al. 2019), consistent with the gamma-ray detection.

Munari \& Walter (2019c) obtained the reddening to the nova from the interstellar features in the optical spectra, deriving $E(B-V)=0.56$, and noted that the comparison with interstellar reddening maps indicates a distance to the nova in excess of $4.5 \mathrm{kpc}$.

In this article, we present the Chandra High Energy Transmission Gratings (HETG) X-ray spectrum observed on the seventh day after the onset of the optical outburst, as illustrated in Figure 1, which allowed us to observe the hard $\mathrm{X}$-ray emission from the ejecta. In Figure 1 we indicate the date of the Chandra observation to place it in the context of the nova evolution. We note that the hydrogen-burning WD was observed as a luminous supersoft X-ray source (SSS) only shortly later, 8.4 days after the outburst on 2019 September 5.25 , when the ejecta had become transparent to soft X-rays (Page et al. 2019). We note that an additional high-resolution X-ray spectrum was obtained with XMM-Newton for the supersoft X-ray phase (Ness et al. 2019) and is the subject of another article that is currently being prepared.

In Section 2 we describe the data we obtained; Section 3 contains an analysis of the spectrum with proposed models for fitting the data; in Section 4 we compare the Chandra HETG spectra observed for two of the other symbiotic RNe and for a classical nova, V959 Mon. They were all observed at a similar evolutionary post-outburst phase. We also briefly discuss what the upper limits on quiescent X-ray emission imply for the cooling time of the ejecta and the secular evolution of the binary. Section 5 contains our conclusions. 


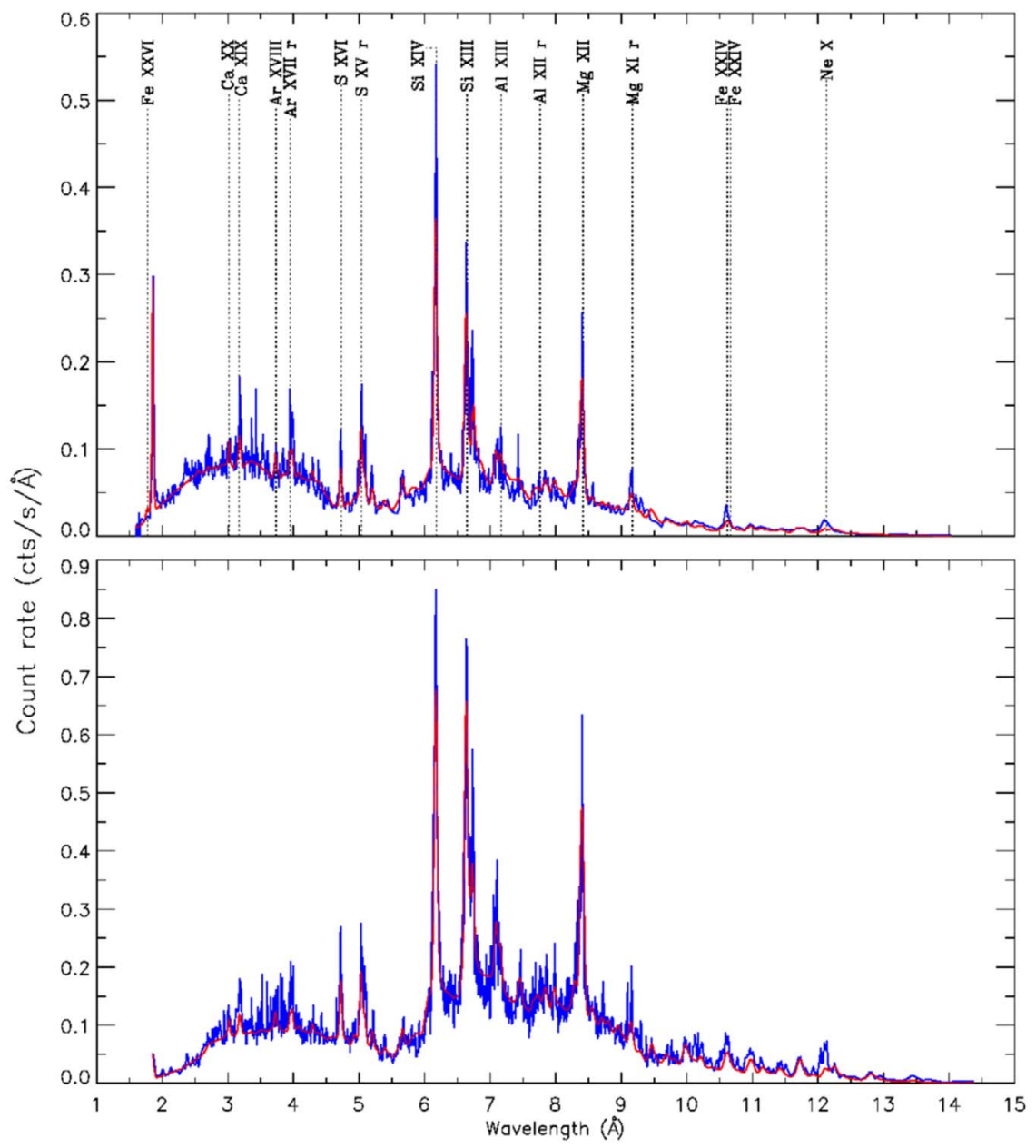

Figure 2. The HEG spectrum is plotted in the upper and the MEG in the lower panel. Both show the wavelength as a function of the count rate, and the strong lines are marked in the HEG panel. The best fit with two APEC thermal components (Model 1) is traced in red in both panels.

\section{The Observed Spectrum}

The Chandra exposure of V3890 Sgr was performed on 2019 September 3 (starting 6.4 days after the initial detection at optical wavelength) with the ACIS-S camera and the HETG. Both the medium-energy grating MEG and the high-energy grating HEG were used, with a respective absolute wavelength accuracy of 0.0006 and $0.011 \AA$. The exposure time was $30 \mathrm{ks}$, and we measured a count rate $0.2139 \pm 0.0027 \mathrm{cts} \mathrm{s}^{-1}$ in the zeroth-order ACIS-S camera. Half of the incident radiation is dispersed to the gratings in this observation mode, and the count rates were $0.5378 \pm 0.0061 \mathrm{cts} \mathrm{s}^{-1}$ in the HEG summed first orders (energy range $0.8-10 \mathrm{keV}$ ) and $1.0450 \pm 0.0063$ in the MEG summed first orders (energy range $0.4-10 \mathrm{keV}$ ). We extracted the spectra with the CIAO software (Fruscione et al. 2006) version 4.9.1 and the CALDB calibration package version 4.8.3. We integrated the flux in the ranges where most of the flux is and the value of signal-to-noise ratio $(\mathrm{S} / \mathrm{N})$ is high, obtaining $1.01 \times 10^{-10} \mathrm{erg} \mathrm{cm}^{-2} \mathrm{~s}^{-1}$ in the $1.5-14 \AA$ range $(0.89-8.27 \mathrm{keV})$ for the HEG, and $1.03 \times 10^{-10} \mathrm{erg} \mathrm{cm}^{-2} \mathrm{~s}^{-1}$ in the $1.8-16 \AA(0.75-6.89 \mathrm{keV})$ range for the MEG. Spectral fitting was done with the HEASOFT XSPEC tool, version 12.6, after the data were binned by $\mathrm{S} / \mathrm{N}$ with the GRPPHA tool (see Dorman \& Arnaud 2001, and references therein).

The HEG and MEG spectra are shown in Figure 2; the prominent emission lines are marked on the HEG spectrum.
Here we adopted the count rate on the $y$-axis for comparison with Drake et al. (2016) and other papers with HETG figures in the literature that use these units. The two spectra are in perfect agreement when plotted with units of flux in the $y$-axis, which we use in the next figures. We note prominent $\mathrm{H}$-like and Helike lines of elements ranging from $\mathrm{Ne}$ to $\mathrm{Fe}$, and some additional Fe-L shell lines.

\section{Spectral Fit and Interpretation}

A thermal plasma model with a single temperature is not sufficient for a statistically acceptable fit, but Figure 2 also shows the best and statistically acceptable spectral fit obtained with two BVAPEC components of thermal plasma in collisional ionization equilibrium (CIE; Astrophysical Plasma Emission Code, or APEC, with variable abundances and velocity broadening in XSPEC, see Smith et al. 2001, and references therein). We used the TBABS prescription for the absorbing equivalent column density of hydrogen atoms (Wilms et al. 2000) and the solar abundances listed in Asplund et al. (2009). We found that $\chi^{2}$ statistics were suitable to assess the goodness of the fit. The parameters of two models and the reduced $\chi^{2}$ value are given in Table 2 . In both cases, the line components are modeled as blueshifted by several hundred $\mathrm{km} \mathrm{s}^{-1}$ and broadened. We constrained the broadening velocity of the cooler component not to exceed that of the hotter component because we assume that the broadening 
Table 2

Parameters of the Best Fits with Two Thermal Components, and Reduced $\chi^{2}$ Value

\begin{tabular}{ll}
\hline \hline Parameter & Value \\
\hline Solar Abundances & \\
\hline$N(\mathrm{H})\left(\mathrm{cm}^{-2}\right)$ & $1.32 \pm 0.05 \times 10^{22}$ \\
$\left.T_{1}(\mathrm{keV})^{-1}\right)$ & $0.99 \pm 0.02$ \\
$v_{1}\left(\mathrm{~km} \mathrm{~s}^{-1}\right)$ & $738 \pm 90$ \\
$\Delta v_{1}\left(\mathrm{~km} \mathrm{~s}^{-1}\right)$ & $1023 \pm 45$ \\
$\mathrm{EM}_{1}\left(\mathrm{~cm}^{3}\right)$ & $8.58 \pm 0.44 \times 10^{56} d(\mathrm{kpc})^{2}$ \\
Flux $_{1, \text { abs }}\left(\mathrm{erg} \mathrm{cm}^{-2} \mathrm{~s}^{-1}\right)$ & $2.56 \pm 0.13 \times 10^{-11}$ \\
Flux $_{1, \mathrm{unabs}}\left(\mathrm{erg} \mathrm{cm}^{-2} \mathrm{~s}^{-1}\right)$ & $1.26 \pm 0.06 \times 10^{-10}$ \\
$T_{2}(\mathrm{keV}$ & $3.99_{-0.20}^{+0.22}$ \\
$v_{2}\left(\mathrm{~km} \mathrm{~s}^{-1}\right)$ & $-522_{-54}^{+42}$ \\
$\Delta v_{2}\left(\mathrm{~km} \mathrm{~s}^{-1}\right)$ & $1023 \pm 45$ \\
$\operatorname{EM}_{2}\left(\mathrm{~cm}^{3}\right)$ & $9.55 \pm 0.50 \times 10^{56} d(\mathrm{kpc})^{2}$ \\
$\operatorname{Flux}_{2, \text { abs }}\left(\mathrm{erg} \mathrm{cm}^{-2} \mathrm{~s}^{-1}\right)$ & $7.79 \pm 0.04 \times 10^{-11}$ \\
$\operatorname{Flux}_{2, \text { unabs }}\left(\mathrm{erg} \mathrm{cm}^{-2} \mathrm{~s}^{-1}\right)$ & $1.36 \pm 0.07 \times 10^{-10}$ \\
$\chi^{2}$ & 1.28 \\
\hline
\end{tabular}

Variable Abundances

\begin{tabular}{ll}
\hline$N(\mathrm{H})\left(\mathrm{cm}^{-2}\right)$ & $8.3_{-1.5}^{+1.1} \times 10^{21}$ \\
$T_{1}(\mathrm{keV})$ & $0.97 \pm 0.03$ \\
$v_{1}\left(\mathrm{~km} \mathrm{~s}^{-1}\right)$ & $-867_{-81}^{+66}$ \\
$\Delta v_{1}\left(\mathrm{~km} \mathrm{~s}^{-1}\right)$ & $632_{-124}^{+146}$ \\
$\mathrm{EM}_{1}\left(\mathrm{~cm}^{3}\right)$ & $0.06_{-0.06}^{+0.50} \times 10^{56} \mathrm{~d}(\mathrm{kpc})^{2}$ \\
Flux $_{1, \text { abs }}\left(\mathrm{erg} \mathrm{cm}^{-2} \mathrm{~s}^{-1}\right)$ & $1.98_{-1.98}^{+16.50} \times 10^{-11}$ \\
Flux $_{1, \text { unabs }}\left(\mathrm{erg} \mathrm{cm}^{-2} \mathrm{~s}^{-1}\right)$ & $6.20_{-6.20}^{+51.67} \times 10^{-11}$ \\
$T_{2}(\mathrm{keV})$ & $4.06_{-0.23}^{+0.25}$ \\
$v_{2}\left(\mathrm{~km} \mathrm{~s}^{-1}\right)$ & $-794_{-180}^{+150}$ \\
$\Delta v_{2}\left(\mathrm{~km} \mathrm{~s}^{-1}\right)$ & $1572_{-72}^{+180}$ \\
$\operatorname{EM}_{2}\left(\mathrm{~cm}^{3}\right)$ & $9.7_{-0.5}^{+0.6} \times 10^{56} d\left(\mathrm{kpc}^{2}\right.$ \\
$\operatorname{Flux}_{2, \text { abs }}\left(\mathrm{erg} \mathrm{cm}^{-2} \mathrm{~s}^{-1}\right)$ & $8.77 \pm 0.90 \times 10^{-11}$ \\
Flux $_{2, \text { unabs }}\left(\mathrm{erg} \mathrm{cm}^{-2} \mathrm{~s}^{-1}\right)$ & $1.42 \pm 0.16 \times 10^{-10}$ \\
$\chi^{2}$ & 1.12
\end{tabular}

Abundances

\begin{tabular}{|c|c|}
\hline$\left[\mathrm{Ne} / \mathrm{Ne}_{\odot}\right]$ & $21_{-21}^{+143}-10.0_{-10.0}^{+3.0}$ \\
\hline $\mathrm{Mg} / \mathrm{Mg}_{\odot}$ & $141_{-133}^{+160}-1.0 \pm 1$ \\
\hline $\mathrm{Al} / \mathrm{Al}_{\odot}$ & $172_{-63}^{+153}-0.0^{+1.0}$ \\
\hline $\mathrm{Si} / \mathrm{Si}_{\odot}$ & $171_{-75}^{+89}-1.5 \pm 1.3$ \\
\hline $\mathrm{S} / \mathrm{S}_{\odot}$ & $236_{-141}^{+319}-1.8 \pm 0.3$ \\
\hline $\mathrm{Ar} / \mathrm{Ar}_{\odot}$ & $411 \pm 232-1.6 \pm 0.6$ \\
\hline $\mathrm{Ca} / \mathrm{Ca} \odot$ & $969_{-500}^{+21}-1.6_{-0.6}^{+0.6}$ \\
\hline $\mathrm{Fe} / \mathrm{Fe}_{\odot}$ & $110_{-96}^{+146}-1.2 \pm 0.14$ \\
\hline
\end{tabular}

Note. The abundance values are given in the text. The $90 \%$ confidence level errors are given, but in the case of the variable abundances after they were calculated after the best-fit abundances were fixed, and for the unabsorbed fluxes after also fixing $N(\mathrm{H})$.

indicates turbulence. We note that this constraint yields the same best-fit $\chi^{2}$ value as when the broadening velocity is allowed to vary independently for the two components (and the broadening velocity of the cooler component turns out to be higher). In the first model, we also constrained all abundances to solar values. The two components of the fit are at $0.99 \mathrm{keV}$ and $3.99 \mathrm{keV}$, respectively. The column density is $N(\mathrm{H})=1.32 \times 10^{22} \mathrm{~cm}^{-3}$. The total flux is overestimated in this fit by about $5 \%$, and the unabsorbed flux at the source is calculated to be $2.62 \times 10^{-10} \mathrm{erg} \mathrm{cm}^{2} \mathrm{~s}^{-1}$. This fit, shown in Figure 2, underestimates the flux in several lines, especially
He-like ones, albeit only by a small amount. However, we could not obtain a better fit by adding more components at different temperature.

In the second model in Table 2, we kept two components, but allowed all the abundances of elements with significant emission lines in the spectrum, that is, $\mathrm{Ne}, \mathrm{Mg}, \mathrm{Al}, \mathrm{Si}, \mathrm{S}, \mathrm{Ar}$, $\mathrm{Ca}$, and $\mathrm{Fe}$, to vary independently from each other, and also independently for each component. However, the cooler component, which may contribute much less than the hot component to the continuum, but has to account for at least four emission lines for each element (H-like and He-like triplets), is largely unconstrained in the fit with all these parameters. The $90 \%$ statistical uncertainties of the emission measure and abundances are very large without any a priori assumptions; our data are not sufficiently good for so many free parameters. Nevertheless, in Table 2 we also give the best-fit parameters we obtained with this second model, again constraining the broadening velocity of the cooler component not to exceed that of the hotter component. The flux was overestimated by almost $8 \%$, but we obtained a lower value of $\chi^{2}$ per degrees of freedom. The two components are at temperatures close to those of the previous case, $0.97 \mathrm{keV}$ and $4.06 \mathrm{keV}$, respectively. This best fit is obtained with very enhanced abundances for the cooler component, and with very high absorption. We obtain instead about nearly solar abundances for the hotter component, except for Al, which turns out to be very depleted. The best-fit value of $N(\mathrm{H})$ is lower than in the previous model, $8.3 \times 10^{21} \mathrm{~cm}^{-3}$, and the unabsorbed flux is $2.04 \times 10^{-10} \mathrm{erg} \mathrm{cm}^{-2} \mathrm{~s}^{-1}$. We stress, however, that given the large uncertainties, the only clear conclusion is that the fit improves when we assume that the cool component has enhanced metal abundances, and that the hot component is consistent with near-solar values.

We came to the conclusion that a CIE is a suitable model by examining helium-like triplets in the spectrum. For each ion, the flux in the " $r$ " recombination line, in the " $i$ " intercombination line and " $f$ " forbidden line gives the so-called $G$ ratio, $G=(f+i) / r$, which indicates whether the plasma is in CIE. Generally, $G>4$ indicates a contribution of photoionization (Gabriel \& Jordan 1969; Bautista \& Kallman 2000; Porquet et al. 2010) as long as this diagnostic is used in a regime where the forbidden line is not sensitive to the density, that is, at extremely high densities (compatible with values of electron density $n_{\mathrm{e}} \geqslant 10^{10} \mathrm{~cm}^{-3}$ in this early post-maximum phase, a value that was inferred at optical wavelengths by Neff et al. (1978, among others). As Figure 3 shows for the Si triplet, the He-like lines were detected with relatively low $\mathrm{S} / \mathrm{N}$, so that the intercombination line is barely observable. It also overlaps with the $r$ and $f$ line, hindering a precise measurement. We thus estimated the $G$ ratio with a fit of three Gaussians to the three lines, tying their broadening velocity to the same value. An example is shown in Figure 3. The resulting flux estimates and their statistical uncertainties are reported in Table 3. Using the flux values reported in the table, we calculated $G=0.94_{-0.38}^{+0.45}$ for S XV, $G=1.21_{-0.35}^{+0.16}$ for Si XIII, $G=0.88_{-0.29}^{+0.34}$ for Mg XI, and $G=0.73_{-0.60}^{+0.89}$ for $\mathrm{Na} \mathrm{X}$ (the statistical uncertainty is given at the $90 \%$ confidence level). Even though our uncertainties are relatively high, these $G$ values are all compatible with a plasma in CIE.

The fluxes given in Table 3 are for lines that could be measured with some precision after subtracting the continuum flux from a nearby region with no emission lines. We can 


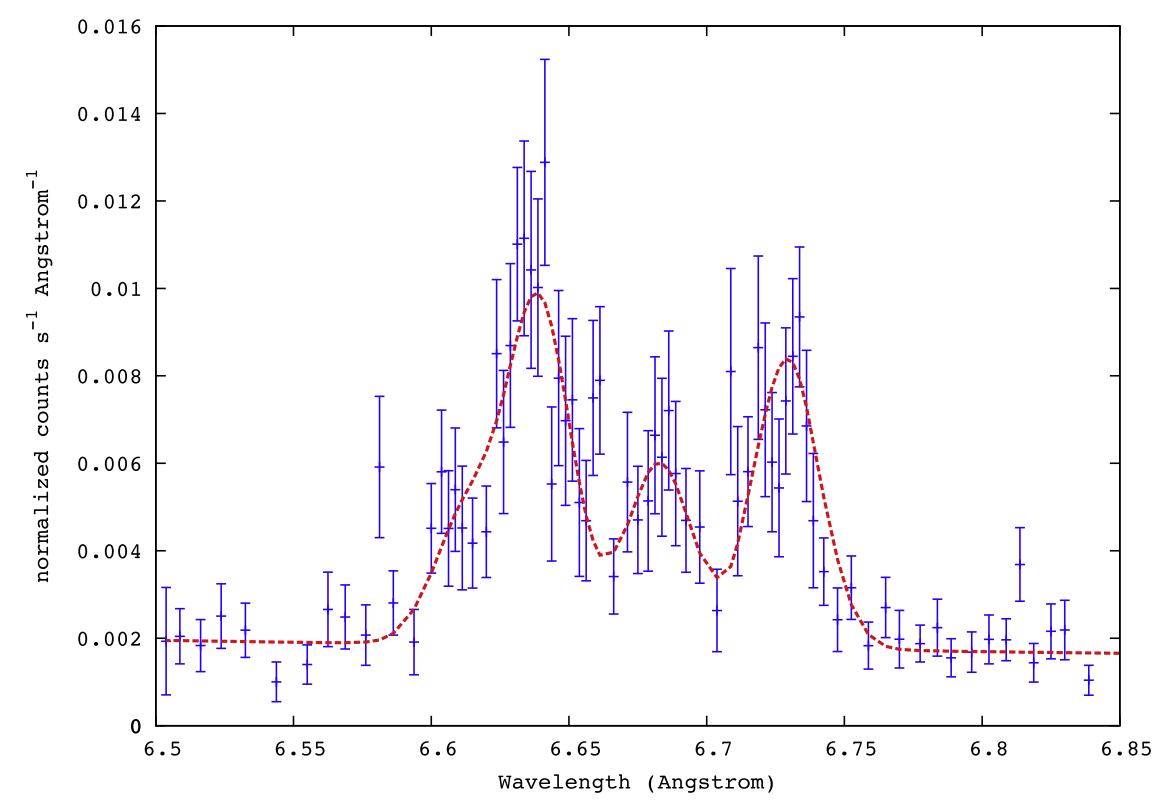

Figure 3. The triplet of Si XIII plotted binned by only 3, thus bringing out the $i$ line, and a possible fit with three Gaussians, plus an additional line blending with the $r$ line (this would be either a line of Al XIII at $6.63 \AA$, or more likely, an additional high-velocity component of the $r$ line). The $y$-axis is in units of photon flux. We did not fit a likely additional emission line at $\approx 6.58 \AA$, sufficiently detached from the triplet.

Table 3

Flux of the Emission Lines

\begin{tabular}{lccc}
\hline \hline Ion & $\begin{array}{c}\text { Rest } \lambda \\
(\AA)\end{array}$ & $\begin{array}{c}\text { Observed } \lambda \\
(\AA)\end{array}$ & $\begin{array}{c}\text { Flux } \times 10^{-13} \\
\left(\mathrm{erg} \mathrm{cm}^{-2} \mathrm{~s}^{-1}\right)\end{array}$ \\
\hline S XVI & 4.727 & 4.720 & $9.23 \pm 3.15$ \\
Si XIV & 6.180 & 6.176 & $15.64_{-1.81}^{+1.91}$ \\
Al XIII & 7.171 & 7.165 & $1.18 \pm 0.59$ \\
Mg XII & 8.419 & 8.412 & $6.21 \pm 0.47$ \\
Ne X & 12.123 & 12.119 & $5.4_{-2.16}^{+0.97}$ \\
S XV $r$ & 5.039 & 5.032 & $7.73_{-1.82}^{+2.07}$ \\
S XV $i$ & $5.063 / 5.067$ & & $2.12_{-1.09}^{+0.73}$ \\
S XV $f$ & 5.10 & 5.101 & $5.14_{-1.99}^{+2.83}$ \\
Si XIII $r$ & 6.648 & 6.639 & $7.71_{-1.96}^{+0.32}$ \\
Si XIII $i$ & $6.685 / 6.688$ & & $4.13_{-0.79}^{+0.85}$ \\
Si XIII $f$ & 6.740 & 6.730 & $5.19_{-0.96}^{+0.80}$ \\
Mg XI $r$ & 9.169 & 9.155 & $2.36_{-0.63}^{+0.74}$ \\
Mg XI $i$ & $9.228 / 9.231$ & & $1.23_{-0.25}^{+0.27}$ \\
Mg XI $f$ & 9.314 & 9.300 & $0.85_{-0.30}^{+0.36}$ \\
Na X $r$ & 11.003 & 10.954 & $2.06_{-1.08}^{+1.49}$ \\
Na X $i$ & $11.080 / 11.083$ & & $0.68_{-0.68}^{+1.12}$ \\
Na X $f$ & 11.192 & 11.143 & $0.83_{-0.62}^{+0.95}$ \\
\hline
\end{tabular}

Note. The H-like lines and the intercombination line are very closely spaced doublets that cannot be resolved. For the first group, H-like lines, we have obtained the flux by direct integration and subtracted the continuum measured in an adjacent spectral region without lines; for the triplets, for which we could not resolve the intercombination line, we have resorted to fitting three Gaussians with the same width and kept the wavelength values shifted by the same amount for the $r, i$, and $f$ lines.

compare the fluxes with those obtained by Peretz et al. (2016) for V959 Mon, known as a nova on an ONe WD. We find that the ratio of the flux of the lines $\mathrm{Al}, \mathrm{Mg}$, and $\mathrm{Ne}$ (elements expected to be enhanced in novae on ONe WDs), relative to the flux of the Si lines, are much lower than in V959 Mon, despite plasma temperatures of about the same value. The ratio of the flux in the H-line of Si XIV to that of $\mathrm{Al}$ XIII is about 1.3 in
V959 Mon, while is about 13 in V3890 Sgr. For Mg XII, this ratio is about 1 in V959 Mon and 2.5 for V3890 Sgr (Table 1 of the above authors reports fluxes in units of incident photons $\times \mathrm{cm}^{-2} \mathrm{~s}^{-1}$, and in order to compare them with our units of $\mathrm{erg} \mathrm{cm}^{-2} \mathrm{~s}^{-1}$, they have to be divided by the wavelength in $\AA$ and multiplied by a factor of $\approx 2 \times 10^{-8}$ ). We suggest that these very different flux ratios are a strong indication in favor of classifying the WD in V3890 Sgr as a CO rather than an ONe one. This is corroborated by the outburst UV spectra of the symbiotic RN RS Oph (which is very similar to V3890 Sgr in many respects), which also indicate a massive carbon-oxygen white dwarf (CO WD; Mikołajewska \& Shara 2017).

As Figure 4 illustrates for four $\mathrm{H}$-like lines, the lines we measure deviate from a simple Gaussian profile: they are asymmetric and skewed toward the blue. A possible interpretation is that the redshifted (receding material) is more absorbed than the blueshifted material, which is moving toward the observer. The ejecta that are receding with respect to us are observed through more foreground layers of ejected plasma. Such an absorption effect is clearly observed in the emission lines of supernova ejecta that form dust (Gehrz \& Ney 1990), and it has also been found in the spectrum of RS Oph (Nelson et al. 2008; Drake et al. 2009), V745 Sco (Drake et al. 2016; Orlando et al. 2017), and V959 Mon, a classical nova with a large luminosity in hard X-rays, before the supersoft X-ray phase (Peretz et al. 2016). In the high-resolution X-ray grating spectra of RS Oph the apparent blueshift was also found to increase with higher wavelength and lower ionization stage, as expected for absorption, which is stronger at higher wavelength. Figure 4 shows that this effect seems to be the same in V3890 Sgr, but it is less evident than in RS Oph.

Another major cause of the skewed profile, certainly overlapping with the differential absorption effect, is indicated by the global fit presented in Table 2 . In fact, for the H-like lines, which have quite different profiles and strengths from the He-like lines, the complex profile can be attributed to at least two components moving at somewhat different velocity and 


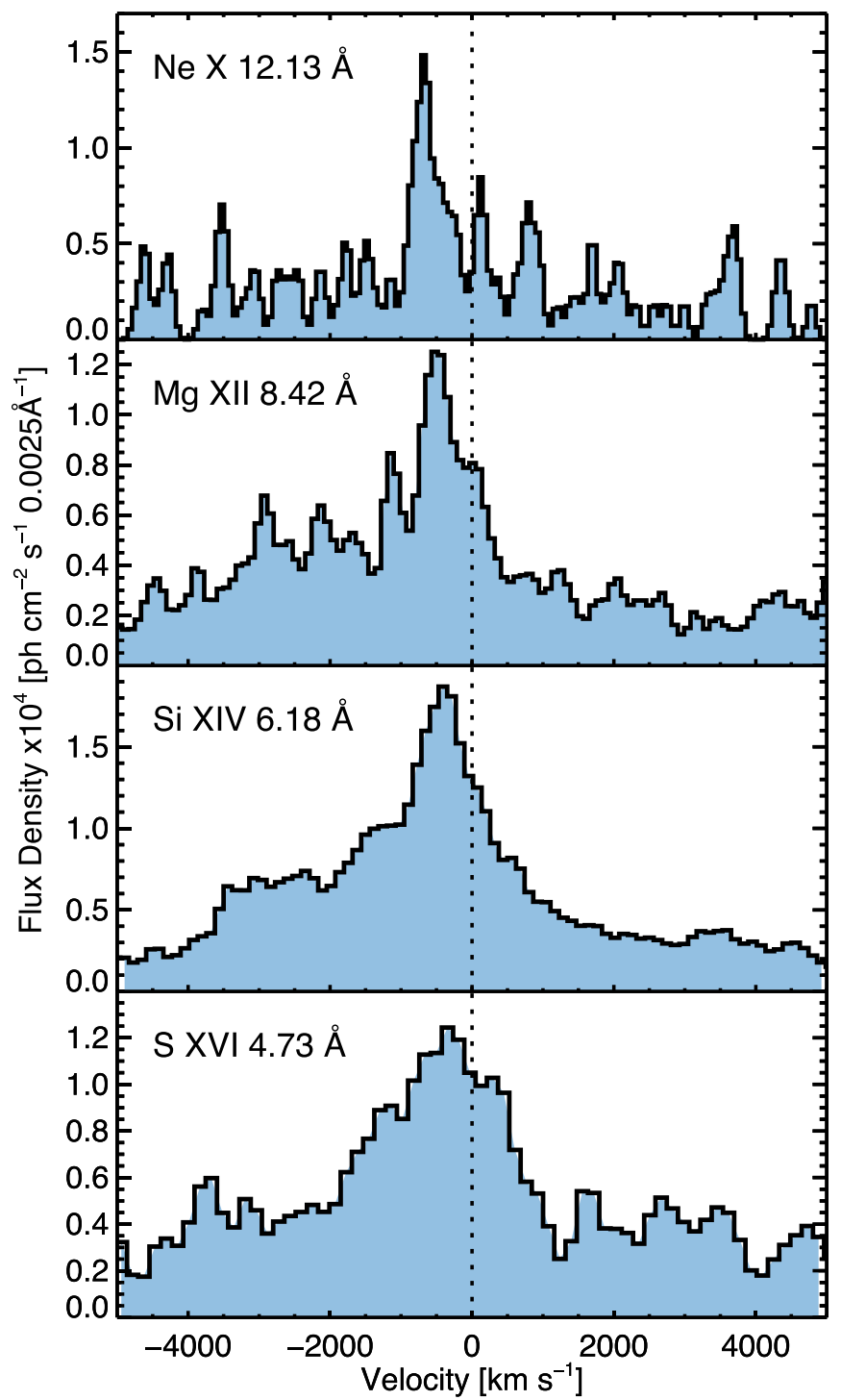

Figure 4. The H-like lines of $\mathrm{Ne} X, \mathrm{Mg}$ XII, Si XIV, and S XVI in the HEG spectrum, plotted in velocity space, showing the asymmetric blueshifted profiles.

with different line broadening. Consistently with the models in Table 2, we interpret this as due to one of the two plasma components that is sufficiently hot to be almost completely ionized. The hot component does not contribute to the flux of the He-like lines, as shown by the line profile and fit in the lower right panel of Figure 5. Unlike the H-like lines, the Helike triplets are fit assuming only the contribution of the cooler component, although we remark that the global model more successfully reproduces the $\mathrm{H}$-like lines than the He-like lines. However, we had to bin the data by $\mathrm{S} / \mathrm{N}$ of at least 20 in order to obtain a statistically significant fit in XSPEC, and with this binning, the weaker $i$ line appears smoothed out, therefore the global fit in XSPEC was made only using the $r$ and $f$ lines.

However, the erosion phenomenon, which is due to differential absorption, makes models with standard software such as those in XSPEC uncertain because the velocities of the two plasma components cannot be precisely estimated without a detailed dynamical model taking into account the effect of the intrinsic nova absorption in the ejecta along the line of sight (e.g., the hydrodynamical model of Orlando et al. 2009, 2017).
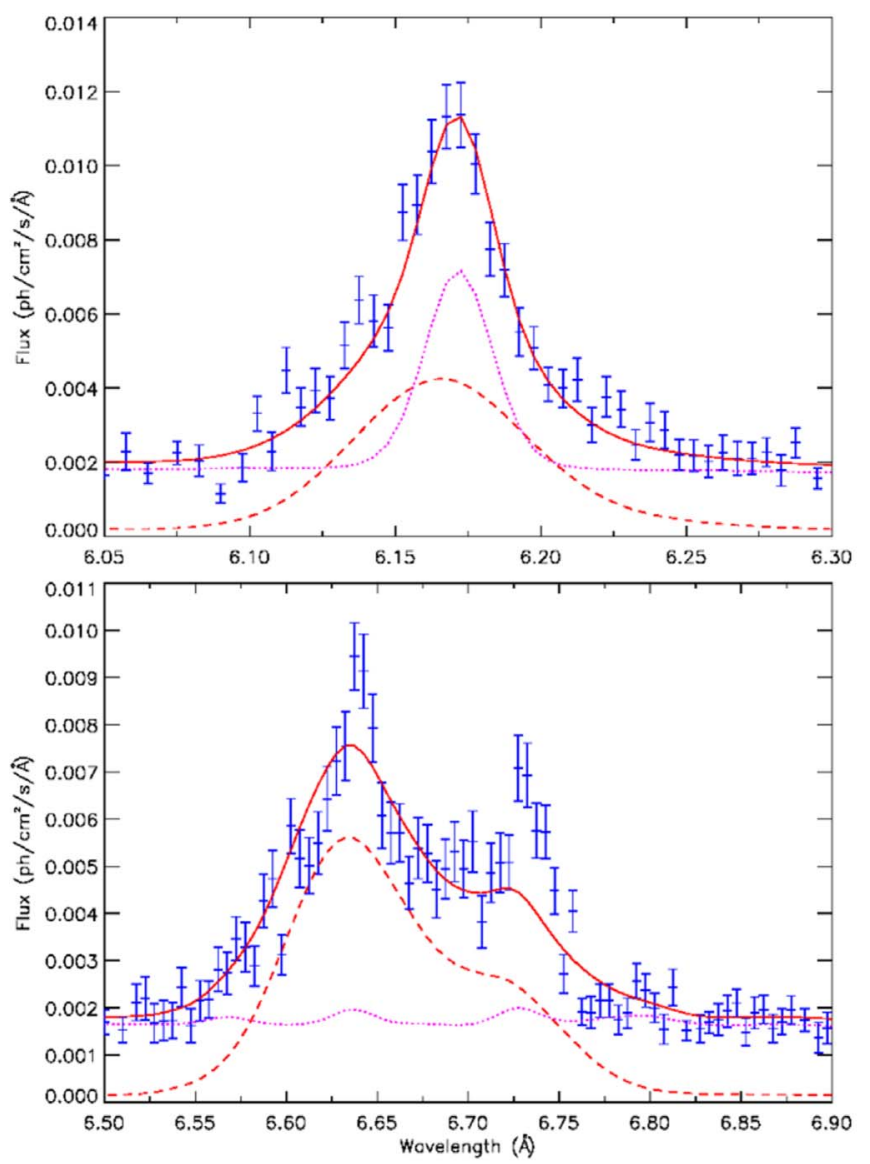

Figure 5. Upper panel: the Si XIV H-like line in the MEG spectrum, binned by $\mathrm{S} / \mathrm{N} \geqslant 20$ as fit with two components with the second model in Table 2 . The dotted pink line shows the hotter component $(\simeq 3.5 \mathrm{keV})$, the dashed red line the $\simeq 1 \mathrm{keV}$ component, and the coadded final model is plotted with a thick solid red line. Lower panel: the same for the Si XIII He-like triplet as observed with the MEG, also with a binning by $\mathrm{S} / \mathrm{N}=20$ : here the hotter component, traced by the dotted pink line, does not contribute to the lines, it only enhances the continuum level.

Despite these uncertainties, it is quite clear that at least two components at different temperature are needed to explain the spectrum of V3890 Sgr, as in the X-ray spectra of the ejecta of other novae previously observed. The first spectrum obtained for V959 Mon in 2012 (but at a much later post-outburst epoch) was fit with two plasma components at about the same temperatures as in Table 2, and the H-like lines indicated the contribution of a hot almost fully ionized component at different velocity than a cooler plasma in which the He-like lines were formed. Mukai et al. (2019) have recently remarked that the two thermal plasma components that are suitable to fit the V959 Mon spectrum may be due to the reverse and forward shock; but the data quality of Peretz et al. (2016) was not sufficient to estimate the velocity of the hotter component. It was consistent with a positive value, versus the negative velocity of the cooler component.

Finally, there may be an undetected supersoft component in V3890 Sgr. Two Swift XRT exposures were performed during the Chandra observation; the first lasted only $450 \mathrm{~s}$ and the second $430 \mathrm{~s}$, but the source was sufficiently bright for spectral fits. 


\subsection{Possibility of a Very Clumpy Medium}

Another diagnostic that can be obtained from the He-like triplets is $R=f / i$, the ratio of the fluxes in the $f$ and $i$ line of each triplet. When this ratio is low, the density is high enough that the collisional deexcitation rate from the upper level of the $f$ transition to the upper level of the $i$ transition is competitive with the radiative decay rate in the $f$ line: the $f$-line emission is thus reduced in favor of the $i$ transitions. With our fit for the $\mathrm{Si}$ XIII triplet, we obtain a value $R=1.26_{-0.33}^{+0.32}$. We also find $R$ values of $2.42_{-1.56}^{+1.57}$ for $\mathrm{S} \mathrm{XV}$, and $0.69_{-0.28}^{+0.33}$ for $\mathrm{Mg}$ XI (the uncertainty represents the $90 \%$ confidence level). For the CIE calculations pertinent to different databases, assuming a negligible radiation field, so that there is no strong UV flux causing radiative photoexcitation increasing the flux in the $i$ line, in Figure 6 we show that the $R$ values we obtain for Si XIII and $\mathrm{Mg} \mathrm{XI}$, and even their lower limits, indicate an electron density higher than a few $n_{e} \times 10^{13} \mathrm{~cm}^{-3}$ for all triplets. While for the $\mathrm{S} \mathrm{XV}$ triplet, the calculated $R$ ratio indicates an electron density value above that examined in the databases, the $90 \%$ confidence level lower limit is consistent with $n_{e} \geqslant 10^{14} \mathrm{~cm}^{-3}$. We could not measure a significant value for $\mathrm{Na} \mathrm{X}$; the error bars are very large.

The electron density above $10^{13} \mathrm{~cm}^{-3}$ appears very high compared with typical electron densities derived from optical spectra, which usually do not exceed $10^{9} \mathrm{~cm}^{-3}$ even at early phases. Neff et al. (1978) estimated a value $n_{e}=1.7 \times 10^{10} \mathrm{~cm}^{-3}$ on the ninth day after the outburst for Nova Cyg 1975 (V1500 Cyg), but they found inhomogeneity in the ejecta and concluded that they were measuring upper limits for this and other parameters. Moore \& Bildsten (2012) calculated a post-shock electron density of the order of $10^{9} \mathrm{~cm}^{-3}$ for the post-shock material in symbiotic recurrent novae assuming a spherically symmetric expansion. The models by Orlando et al. $(2009,2017)$ also imply a post-shock electron density of $10^{9}-10^{10} \mathrm{~cm}^{-3}$. However, we know that in two classical novae, U Sco (Orio 2013) and V959 Mon (Peretz et al. 2016), the high-resolution X-ray spectra imply much higher density at an epoch when the contribution of photoexcitation to the $i$ line was already negligible. Whether this critical assumption on the photoexcitation contribution is also valid for V3890 Sgr depends critically on the WD temperature on the day of observation, and on the distance at which the shocks occurred from the WD.

According to model calculations for the similar symbiotic nova RS Oph (D. Prialnik 2020, private communication, see also Hillman et al. 2014), the photospheric radius of the WD is about $3.5 \times 10^{11} \mathrm{~cm}$ during the whole period before the SSS emerges, then it shrinks to a value around $0.01 R_{\odot}\left(\approx 7 \times 10^{9}\right.$ $\mathrm{cm})$ within less than a day. The effective temperature before the shrinking leading to the SSS is about $30,000 \mathrm{~K}$; it moves to values above $200,000 \mathrm{~K}$ within few hours. In order to assess the importance of photoexcitation, we need to compare its rate with the decay rate of the forbidden line. This is slow, and for Si XIII, it is $3.5 \times 10^{5} \mathrm{~s}^{-1}$. The expression for the photoexcitation rate is $0.026 \times f_{i j} F_{\nu(16 \mathrm{eV})}$, where $f_{i j}=0.054$ is the oscillator strength for the transition between the upper levels of the forbidden and intercombination lines, and $F_{\nu(16 \mathrm{eV})}$ is the UV flux at $16 \mathrm{eV}$ at a given distance from the WD. In our specific case, the conclusion is uncertain because it is not straightforward to evaluate the photospheric effective temperature of the central source in V3890 Sgr at the particular time of our Chandra exposure. In fact, the WD was detected as an SSS
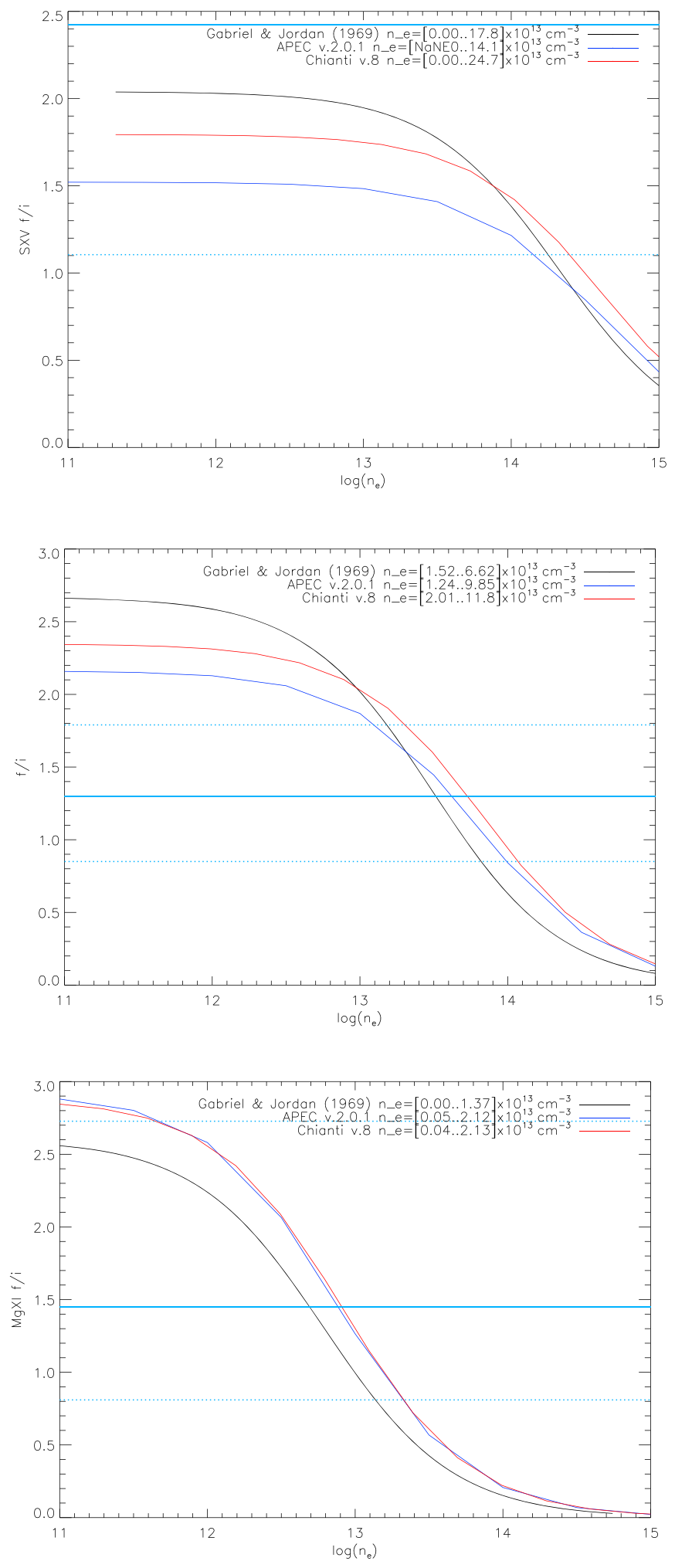

Figure 6. The $f / i$ ratio of the $\mathrm{S} \mathrm{XV}, \mathrm{Si} \mathrm{XIII}$, and $\mathrm{Mg}$ XI triplets as a function of density in absence of strong photoexcitation, according to the Gabriel \& Jordan (1969), APEC (Smith et al. 2001), and Chianti (del Zanna et al. 2015) databases. The solid blue line shows the measured value, and the dotted lines show the lower and upper limit given the $1 \sigma$ uncertainty (only the lower limit for $S X V)$.

only two days later, so the WD radius may already have been shrinking, while the ejecta may still have been largely absorbing the supersoft X-rays. There is a very large difference 
between the luminosity at $16 \mathrm{eV}$ of a blackbody-like source emitting $10^{38} \mathrm{erg} \mathrm{s}^{-1}$ with a temperature of $30,000 \mathrm{~K}$, about $10^{37} \mathrm{erg} \mathrm{s}^{-1}$, and the luminosity at $16 \mathrm{eV}$ of a source with a blackbody temperature above $200,000 \mathrm{~K}$ (as inferred with the Swift XRT observation two days after our spectrum was taken). If our source was already at such a high temperature while the ejecta were not yet quite transparent to the X-rays, the peak of the luminosity must already have been in the X-rays and the $16 \mathrm{eV}$ luminosity would have been negligible.

If instead the effective temperature during our observation was still $30,000 \mathrm{~K}$ and dropped only on the following day, the calculation indicates that the UV flux was still significant. In this case, the distance at which the photoexcitation rate equals the decay rate of the Si XIII forbidden line in au is 17.9 au. In the model of Orlando et al. (2009) for RS Oph, the red giant is at $1.5 \mathrm{au}$ from the WD, and the peak of the flux is at 4 au from the red giant, so the distance from the WD would be smaller, only $5.5 \mathrm{au}$. The photoexcitation rate in such a case is about 10 times higher than the decay rate of the forbidden line. The contribution of the UV source at a distance of a few au would have still been very significant and $n_{e}$ cannot be derived from the ratio of the triplet lines.

It is interesting to note that for $\mathrm{V} 745 \mathrm{Sco}$, the $R$ ratios indicated $n_{e} \leqslant 10^{9} \mathrm{~cm}^{-3}$ (Drake et al. 2009), while in RS Oph, only the $R$ ratio derived from $\mathrm{Mg}$ XI may have been due to an electron density above $10^{10} \mathrm{~cm}^{-3}$, but the error bar was so large that the authors did not comment on it (Nelson et al. 2008). In this respect, the X-ray spectrum of V3890 Sgr differs from the two other symbiotic RNe with similar orbital properties. If the WD photosphere had already moved toward a peak of emission in the extreme UV and was no longer a UV source, the high electron density we derive for V3890 Sgr is comparable to the high values obtained for the classical novae U Sco and V959 Mon.

What are the implications of an electron density of a few $10^{13} \mathrm{~cm}^{-3}$ ? One caveat is the very short radiative cooling time. The ratio of the thermal energy of the plasma to the radiative loss rate (at the plasma temperatures we estimated with the spectral fit) implies a radiative cooling time of only $1 \mathrm{~s}$, instead of several days as in the model of Moore \& Bildsten (2012). This means that there must be a constant supply of ejected mass during all the time this thermal spectrum was measured, including the later and earlier Swift XRT observations. Even with the maximum nova wind velocity estimated to be about $4000 \mathrm{~km} \mathrm{~s}^{-1}$, a spherically symmetric shocked region would be very thin, of about only $4000 \mathrm{~km}$.

The value of the total emission measure $\mathrm{EM}=\int n_{e} n_{\mathrm{i}} d V \approx n_{e} n_{\mathrm{i}} V$ that we obtain by adding the emission measure of the two model components in Table 2 is $\mathrm{EM}=1.81 \times 10^{56} \times d(\mathrm{kpc})^{2} \mathrm{~cm}^{3}$ in the solar abundance model and $\mathrm{EM}=9.76 \times 10^{55} \times d(\mathrm{kpc})^{2} \mathrm{~cm}^{3}$ in the model with variable abundances. Assuming $d=4.4 \mathrm{kpc}$, we find that $\mathrm{EM}=(1.9-3.5) \times 10^{57} \mathrm{~cm}^{-3}$. When we assume that the X-ray emitting ejecta traveled at constant velocity for a week and that they fill a spherical volume $V, V=5.9 \times 10^{43} \mathrm{~cm}^{3}$, our value of the emission measure yields $n_{\mathrm{e}} \approx(6-8) \times 10^{6} \mathrm{~cm}^{-3}$, which is six orders of magnitude smaller than estimated above using the Si XIII triplet, and it cannot be reconciled with a slower expansion velocity or a more refined calculation. We can only conclude that the volume filled by the shocked plasma is a very small fraction of the volume that homogeneous ejecta with a filling factor of 1 would fill.
Following the reasoning of Peretz et al. (2016) and, like these authors, assuming for simplicity a fully ionized gas with $n_{e}=n_{p}$ (where $n_{p}$ is the proton density) and approximately solar composition, the mass of the emitting plasma is

$$
M=X Y m_{p} \frac{\mathrm{EM}}{n_{e}} \approx 11.25 m_{p} \frac{\mathrm{EM}}{n_{e}},
$$

where $X$ is the mean proton number per ion, $X=1.5$, and $Y$ is the mean atomic weight (we assumed $Y=7.5$; see also Peretz 2016) .For $n_{e} \approx 10^{-13} \mathrm{~cm}^{-3}$, a simple calculation indicates that the emitting mass is $M=1.8 \times 10^{-12} M_{\odot}$ assuming a $4.4 \mathrm{kpc}$ distance, which is a very small fraction of $M_{\text {ej }}=1.1 \times 10^{-6} M_{\odot}$ estimated, for instance, for RS Oph by O'Brien et al. (1992; we suggest that the total $M_{\mathrm{ej}}$ is likely to be on the same order for all symbiotic RNe, including V3890 Sgr, see also the model calculations by Yaron et al. 2005). This indicates that the X-ray emitting material is not uniformly distributed, but is rather concentrated in one small region, or in a number of clumps occupying a small volume. We note that for densities on the order assumed by Orlando et al. (2009, 2017), the shocked mass in this observation instead is a few $10^{-8} M_{\odot}$. Very clumpy emitting material was inferred for the nonsymbiotic T Pyx (Tofflemire et al. 2013), U Sco (Orio et al. 2013), and for V959 Mon (Peretz et al. 2016). In Section 5 we also discuss the possibility that the shocks at the stage at which we observed this symbiotic nova occurred in a very small region, namely close to the red giant.

\section{Comparison with the Chandra HETG Spectra of Other Novae}

The known physical parameters of V3890 Sgr have been compared with the other Galactic symbiotic RNe in Table 1. A comparison of our spectra with those obtained in the early phases of other symbiotic RNe outbursts with the same gratings shown in Figure 7 indicates rather remarkable similarities between this nova, the two other recurrent symbiotic novae that were observed with X-ray gratings, and the classical nova V959 Mon, which was also observed before the supersoft $\mathrm{X}$-ray phase, when the central source outshines the spectrum of the ejecta. The HETG spectra of RS Oph were presented by Nelson et al. (2008), Ness et al. (2009), the spectrum of V745 Sco by Drake et al. (2016), and that of V959 Mon by Peretz et al. (2016). Table 4 lists the X-ray flux and the absorbed and unabsorbed X-ray luminosity of these novae when their ejecta were observed with X-ray gratings. V745 Sco had an extremely short SSS phase, and the central source contributed to the X-ray flux copiously on day 10 in the Swift XRT range, so the actual flux from the ejecta is between the value derived from Swift + NuSTAR and NuSTAR alone (3-79 keV range). When it was observed with the Chandra HETG on day 14, the central source had already turned off.

At a distance of $4.4 \mathrm{kpc}$, which is likely to be only a lower limit, V3890 Sgr on day 7 had a high X-ray luminosity, $L_{\mathrm{X}}=1.91 \times 10^{35} \mathrm{erg} \mathrm{s}^{-1}$, and the unabsorbed luminosity was a factor of 2-3 higher. This X-ray luminosity is also comparable to that of RS Oph and V745 Sco. Like the gamma-ray luminosity, the X-ray luminosity due to the shocked ejecta also seems to be much higher for the symbiotic novae than for the short-period ones. Short-period novae in outburst before (or after) the supersoft X-ray phase do not 


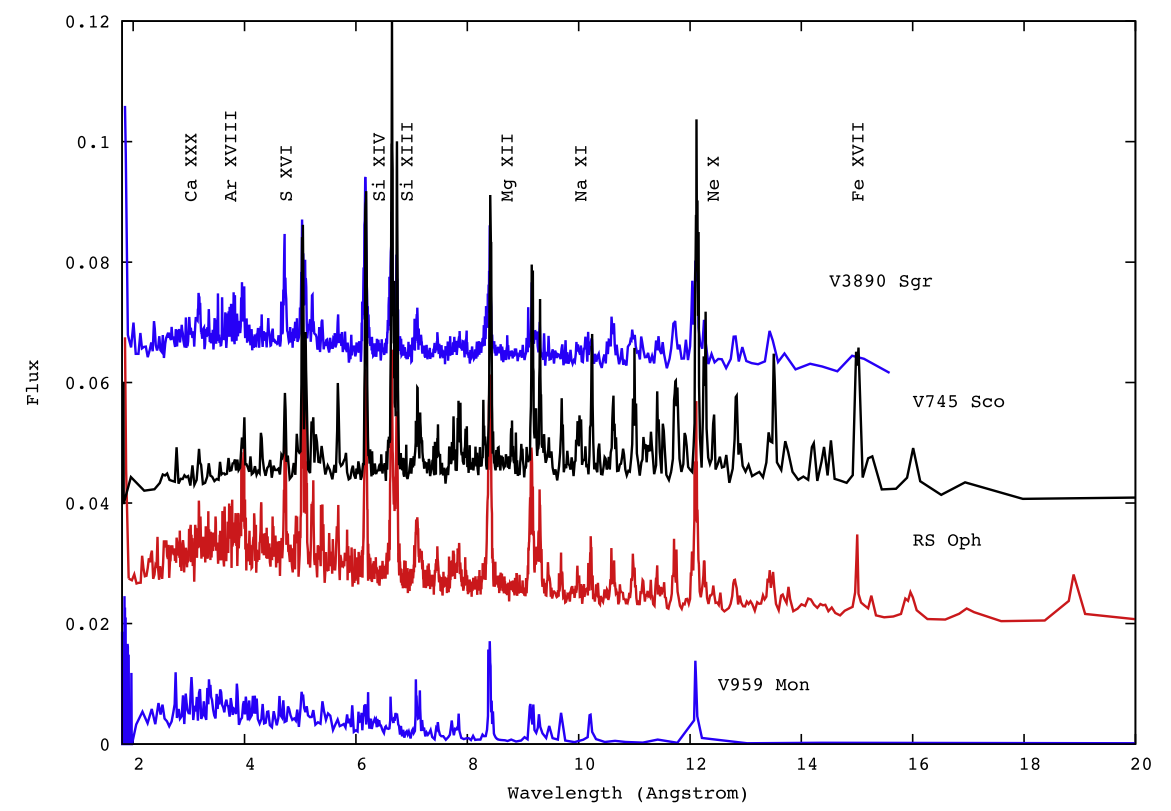

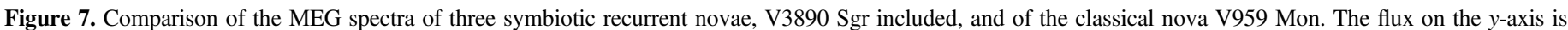

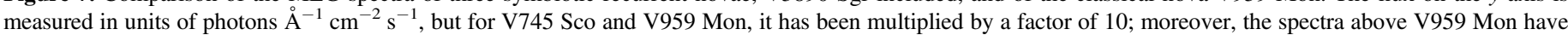
each been offset for clarity, from bottom to top, by 0.02 photons $\AA^{-1} \mathrm{~cm}^{-2} \mathrm{~s}^{-1}$ with respect to the spectrum below it.

exceed an X-ray luminosity of $10^{33} \mathrm{erg} \mathrm{s}^{-1}$ (Orio et al. 2001; Ness et al. 2007). Because symbiotic novae differ from the others because of the red giant companion, this is considered an indication that the impact with the red giant wind must be causing the shocks. An exception is the classical nova V959 Mon, which has a $7 \mathrm{hr}$ orbital period and is not a symbiotic. It exploded when it was close to the Sun and could not be observed until much later, so the outburst day of the HETG observation is an estimate. This nova was discovered as a gamma-ray source with Fermi, and it may have been much more X-ray luminous previously. However, the hard X-ray luminosity about two months after the estimated optical maximum was still very high. If V959 Mon is not an RN (no previous outburst was ever reported), it is an exception among short-period novae. This nova would have had time to accrete a larger envelope than $\mathrm{RNe}$, ejecting a much higher mass that slowed the X-ray flux emergence and evolution, but it is difficult to explain the high X-ray luminosity without a new episode of mass ejection after the initial peak.

The spectra of V745 Sco and RS Oph differ from that of V3890 Sgr in the soft portion, where no significant emission lines of oxygen and nitrogen were measured. The APEC fits to the RS Oph and V745 Sco spectra in fact required more than only two components to explain the observed range of atomic transitions (Nelson et al. 2008; Drake et al. 2016). An important difference between V959 Mon and the symbiotic $\mathrm{RNe}$ are the ratios of lines of different species. The ratios of emission lines that are the strongest in oxygen-neon $(\mathrm{ONe})$ novae, such as V959, indicate nucleosynthesis and elemental abundances that are predicted to be the result of a TNR on an $\mathrm{ONe} \mathrm{WD}$, where $\mathrm{Ne}-\mathrm{Na}$ and $\mathrm{Mg}-\mathrm{Al}$ cycles that do not occur on CO WDs operate in addition to the CNO cycle (José \& Hernanz 1998; Starrfield et al. 2009; Kelly et al. 2013). Atomic $\mathrm{Mg}$ (sum of three isotopes) is also overabundant in a superficial layer on $\mathrm{ONe}$ WDs. The emission lines of $\mathrm{Si}$ and $\mathrm{Fe}$ are stronger than those of $\mathrm{Mg}, \mathrm{Ne}$, and $\mathrm{Al}$ in our spectrum of V3890 Sgr. V959 Mon instead has more abundant flux in the
$\mathrm{Mg}, \mathrm{Ne}$, and $\mathrm{Al}$ lines than in those of $\mathrm{Si}$ and $\mathrm{Fe}$, even though the spectrum was fit with two plasma components at approximately the same temperature that we obtained in this paper for V3890 Sgr (Peretz et al. 2016). When we compare V3890 Sgr to RS Oph and V745 Sco, the line flux ratios of the three symbiotic RNe spectra are instead about the same. Of course, if the material has mixed with the red giant wind, we do not expect to find "clean" signatures of the ashes burned on the WD, so a conclusion on the WD nature (whether it is of CO or $\mathrm{ONe}$ ) does not always seem feasible for symbiotic $\mathrm{RNe}$ on the basis of the X-ray emitted in the nova outflow, but the difference with the V959 Mon spectrum is rather striking.

\subsection{Cooling Time and X-Ray Flux at Quiescence}

A discussion of the cooling time of the ejecta in postoutburst symbiotic RNe is found in Moore \& Bildsten (2012). These authors assume a spherical explosion and that shocks typically occur at a distance of a few au from the red giant. They discuss the implications for a symbiotic RN ending its life in an SN Ia explosion. X-ray flux after the RN outburst should only be detectable for a cooling time that was found to vary from a few days to a few weeks. It is predicted to be about two weeks for RS Oph, and after this time, the kinematics of the ejecta are dominated by momentum (rather than energy) conservation.

Sokoloski et al. (2006) observed that the X-ray flux from the ejecta of RS Oph was related to the time $t$ in days since maximum as $t^{-5 / 3}$. The Swift XRT archival observations of V3890 Sgr show that V3890 Sgr cooled much more slowly than RS Oph because the flux on 2019 November 19 was still $6.7 \times 10^{-12} \mathrm{erg} \mathrm{cm}^{-2} \mathrm{~s}^{-1}$ in the $0.3-10 \mathrm{keV}$ range of the Swift XRT.

Another interesting measure is the X-ray flux at quiescence, and whether it originates in the boundary layer of an accretion disk and gives a measure of the accretion rate. After the 1990 outburst, V3890 Sgr was still detected in X-rays in the short ROSAT All-Sky-Survey exposures (Orio 1993) about 5 
Table 4

X-Ray Emission of the Ejecta of Three Symbiotic RNe and V959 Mon

\begin{tabular}{|c|c|c|c|c|}
\hline Nova & Day & $\begin{array}{c}\text { Flux } \\
\left(\text { erg } \mathrm{cm}^{-2} \mathrm{~s}^{-1}\right)\end{array}$ & $\begin{array}{l}\text { Luminosity } \\
\left(\mathrm{erg} \mathrm{s}^{-1}\right)\end{array}$ & $\begin{array}{l}\text { Lum. (unabs.) } \\
\quad\left(\mathrm{erg} \mathrm{s}^{-1}\right)\end{array}$ \\
\hline V3890 Sgr & 8 & $1.01 \times 10^{-10}$ & $2.33 \times 10^{35}$ & $4.6-7 \times 10^{35}$ \\
\hline \multirow[t]{2}{*}{ RS Oph } & 7 & $\geqslant 1.98 \times 10^{-9}$ & $\geqslant 7 \times 10^{35}$ & \\
\hline & 14 & $6.09 \times 10^{-10}$ & $1.86 \times 10^{35}$ & \\
\hline \multirow[t]{2}{*}{ V745 Sco } & 10 & $(1.6<F<2.39) \times 10^{-10}$ & $(1.16<L<1.73) \times 10^{36}$ & \\
\hline & 16 & $4.21 \times 10^{-11}$ & $3.05 \times 10^{35}$ & \\
\hline V959 Mon & 83 & $1.7 \times 10^{-11}$ & $2.33 \times 10^{34}$ & \\
\hline
\end{tabular}

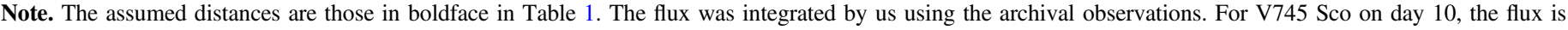

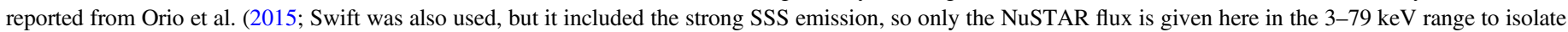

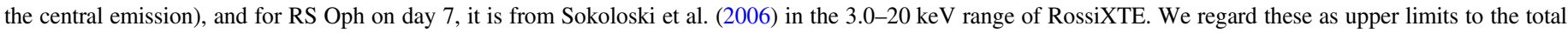
flux of the ejecta. For V959 Mon, the flux is in the $0.3-10 \mathrm{keV}$ band.

months after maximum, but it was no longer detected in serendipitous pointings 11 and 17 months after the outburst (Orio et al. 2001). This is also consistent with an XMMNewton exposure on 2010 April (archival observation by P.I. Sokoloski), in which we found an upper limit for the X-ray luminosity at $4.5 \mathrm{kpc}$ of approximately $10^{31} \mathrm{erg} \mathrm{s}^{-1}$. V745 Sco, for comparison, was only marginally detected four years before the last outburst with XMM-Newton, with an X-ray luminosity $6 \times 10^{31} \mathrm{erg} \mathrm{s}^{-1}$ in the $0.3-8.0 \mathrm{keV}$ range (see Luna et al. 2014).

The X-ray flux of RS Oph at quiescence is variable, and on average, it seems to have increased between 1991-1992 and 2007-2008 (Orio 1993; Orio et al. 2001; Nelson et al. 2011). Orio (1993) found that the X-ray flux measured in 1991 was not consistent with the boundary layer of a disk with high $\dot{m}$, but an observation in 2008 April with Chandra showed an X-ray flux that was marginally consistent with $\dot{m} \geqslant 2 \times 10^{-8}$ $M_{\odot} \mathrm{yr}^{-1}$ (Nelson et al. 2011), explaining the occurrence of outbursts every 10-20 yr with model calculations with the same $\dot{m}$ value (e.g., Yaron et al. 2005).

Only $\mathrm{T}$ CrB, which is at less than $1 \mathrm{kpc}$ distance, emits significant variable $\mathrm{X}$-ray flux at quiescence that can be attributed to accretion (see Luna et al. 2008, 2013, 2018). It is also quite a variable and hard X-ray source, as expected for a disk boundary layer around a massive WD (Iłkiewicz et al. 2016; Luna et al. 2018). T CrB stands out, in comparison with the other symbiotic RNe, with its conspicuous hard X-ray flux at quiescence. It has never been observed in X-rays in outburst, and the next eruption would be an opportunity not to be missed.

\section{Discussion and Conclusions}

On the seventh day after the optical maximum, V3890 Sgr was emitting an X-ray luminosity of (4.6-7) $\times 10^{35} \mathrm{erg} \mathrm{s}^{-1}$ in the $0.7-9 \mathrm{keV}$ range (depending on the absorbing column density, and assuming a distance of $4.5 \mathrm{kpc}$, which is likely a lower limit). We attribute this emission to the plasma outflowing from the nova, and suggest that strong shocks formed when it impacted the circumbinary medium filled by the red giant wind. The same physical explanation has been suggested for other symbiotic novae, which typically emit much higher X-ray flux than short-period systems. The spectrum of V3890 Sgr can be fit with a model of thermal plasma in CIE, and shows prominent asymmetric emission lines of $\mathrm{Mg}, \mathrm{Ne}, \mathrm{Na}, \mathrm{Si}, \mathrm{S}, \mathrm{Ar}, \mathrm{Ca}$, and $\mathrm{Fe}$.

Our analysis provides the following important points:
(1) The lines due to H-like transitions are stronger than the lines due to He-like transitions. This can be explained by at least two thermal plasma components, and in fact we obtain a fit to the spectrum with two components in CIE, at temperatures of approximately $1 \mathrm{keV}$ and $4 \mathrm{keV}$, respectively. The hotter plasma is almost completely ionized and does not contribute to the emission lines due to He-like transitions. A possible interpretation is that the two components represent a forward and a reverse shock.

(2) An alternative interpretation can be suggested by analogy with a model for RS Oph of Orlando et al. (2009): the two plasma components may represent colder material that is not much mixed with the red giant wind, and hotter material that is instead heavily mixed and has been heated, respectively. In our model fit, there is in fact some evidence that the cooler plasma has enhanced metal abundances with respect to the hotter component, resembling two regions in the hydrodynamical model of Orlando et al. (2009) for RS Oph, which predicts that the zones containing mostly ejecta material are denser and colder, while in regions where shocked ejecta have mixed with circumstellar material, the plasma is hotter and dominated by thermal conduction. For RS Oph, the authors were able to model the He-like resonance line profiles with the overlap of the two components, in a similar manner as in our Figure 5 for the H-like lines. When this interpretation is applied to V3890 Sgr, the higher abundances of the cold region indicated by the model with free abundances may be due to nova nucleosynthesis products in the ejecta. The hotter mixed ejecta on day 5 for RS Oph would have contributed $80 \%$ of the observed flux, which is similar to our finding that the hotter plasma for V3890 Sgr on day 6 contributed to at least $70 \%$ of the observed flux.

(3) Our APEC spectral model in XSPEC fits the data, but it is approximate and phenomenological, while only a detailed hydrodynamical model may quantify the effect of the differential absorption and distinguish the exact contribution of the two (or possibly more) different plasma regions to the line profiles. One reason why the model is still too simplistic is that we have only one absorbing component with characteristics corresponding to the interstellar medium along the line of sight. Instead, there is also significant intrinsic absorption of the nova ejecta (in fact, the column density was observed with the Swift XRT to decrease as the ejecta expanded; Page et al. 2020, in preparation). We suggest that only a detailed 
hydrodynamical model may explain the formation of the different emission lines in a rigorous way and account for profile and flux of each single line.

(4) The X-ray spectrum of V3890 Sgr differs from that of the two symbiotic RNe with similar orbital parameters, RS Oph and V745 Sco, in the line ratios of the He-like triplets. The $R=f / i$ line ratios are quite lower than those in the spectra of the two previous symbiotic RN. The values we evaluated indicate high electron density (a few $10^{13} \mathrm{~cm}^{-3}$ ), unless there was still significant UV flux from the photosphere. Unfortunately, at the epoch the spectrum was taken, it is difficult to establish whether the emission of the WD photosphere was already peaking in the extreme UV or in the supersoft X-rays. If the density was indeed very high, the emission must have arisen from very clumpy ejecta constituting only a small fraction of the emitted material, of the order of $10^{-12} M_{\odot}$. This is not a completely unusual and new scenario; in fact, similarly high electron density has been inferred for other novae (Orio 2013; Tofflemire et al. 2013; Peretz et al. 2016), suggesting that in some novae, the shocked material that emits X-rays may be emitted as dense "bullets," in a clumpy rather than smooth outflow.

(5) There are interesting caveats relative to the possibility of very high electron density. First of all, the cooling time of the shock would be much shorter than in the models of Orlando et al. (2009) and Moore \& Bildsten (2012) for RS Oph. It would be only about $1 \mathrm{~s}$, so the shock must be continuously powered, requiring a constant mass outflow for a prolonged time. Second, and most important, in case of nonradiative shocks, the density ratio of pre- and postshock material should be about 4 . Even taking into account radiative cooling, the density gradient cannot have been as large as orders of magnitude, but the typical red giant wind electron density at $\simeq 4$ au from the red giant (where Orlando et al. 2009 estimate that the shocks occurred in the RS Oph case) is only $10^{9} \mathrm{~cm}^{-3}$ (see the analytical formula in Moore \& Bildsten 2012). Even with the proposed equatorial density enhancement, the density is only approximately $10^{10} \mathrm{~cm}^{-3}$. Because the density scales with the square of the distance from the red giant, perhaps the shocks in V3890 Sgr did not occur at a distance of a few au from the red giant, but the site of $\mathrm{X}$-ray emission was instead very close to the giant's photosphere. In this case, the emitting region subtended a small solid angle and occupied a small volume.

With a spectrum taken at only one epoch, we cannot distinguish between the two possibilities: emission originating at distance from the star of a few au, which implies that the $R$ line ratio is the product of a strong photoionizing source, and an actual electron density of the order of $10^{13} \mathrm{~cm}^{-3}$. We know that two days after our spectrum was taken, the photoionizing flux was negligible, but the only other high-resolution X-ray spectrum of V3890 Sgr was obtained only 11 days later (Ness et al. 2019, and J.U. Ness et al. 2020, in preparation), and unfortunately, this second spectrum was taken too late. Although at this epoch the central SSS emitted negligible UV flux and did not photoexcite the $i$ line, the flux had decreased, and measurements of the $R$ ratios were not feasible. This highlights the fact that in a fast symbiotic nova like V3890 Sgr, the cadence of the spectral exposures should be evaluated carefully in the future: multiple exposures, close in time, would be very useful. A second spectrum taken only few days later, when the central source was peaking in the X-rays and clearly no longer contributed significant UV flux, might have solved the conundrum.

(6) Even though we have highlighted the intriguing possibility that the V3890 Sgr spectrum may have originated in shocks within a small volume of dense emitting plasma close to the red giant photosphere instead of a site at a distance of a few au from the red giant, like in RS Oph and V745 Sco, the X-ray luminosity of V3890 Sgr is in the same range as observed for the two other symbiotic $\mathrm{RNe}$ that are observed in hard X-rays in the two weeks following the optical maximum, and it is about two orders of magnitude higher than in most short-period classical novae. Another symbiotic nova, V407 Cyg, not known to be recurrent, also had very X-ray luminous ejecta (Orlando \& Drake 2012). Only the short-period nova V959 Mon was observed to have an X-ray luminosity of a few $10^{34} \mathrm{erg} \mathrm{s}^{-1}$ three months after optical maximum; all other short-period novae have much less luminous X-ray ejecta. The early X-ray spectra of all three symbiotic $\mathrm{RN}$ observed so far with the $\mathrm{X}$-ray gratings show remarkable similarities not only in the luminosity level, but also in line profiles, and (from initial estimates based on the line fluxes) probably in the chemical abundances of the emitting material.

(7) The observed thermal X-ray emission cannot be directly linked to the measured gamma-ray flux for this nova. The gamma-rays would only originate in the initially X-ray shocked plasma if the thermal plasma were much hotter, and if there were also the contribution of a nonthermal component to the X-ray flux (Metzger et al. 2015). However, we would like to note that the possibility of a dense and clumpy plasma evoked by the high electron density gives some support to the idea that possible initial shocks giving rise to the gamma-rays may also be difficult to observe because they would easily be "buried" within a large volume of non-X-ray emitting absorbing material (as suggested by Nelson et al. 2019).

(8) The relative ratios of the flux in $\mathrm{Al}, \mathrm{Mg}$, and $\mathrm{Ne}$ lines (typical of novae on ONe WDs) to the flux in lines of $\mathrm{Si}$ and $\mathrm{Fe}$ (which are not specifically related to ONe WDs) in the spectrum of V3890 Sgr, like in the other symbiotic RNe RS Oph and V745 Sco, are much lower than in the known ONe short-period nova, V959 Mon. This suggests that V3890 Sgr hosts a CO WD.

In the future, obtaining high-resolution emission line spectra of the ejecta of symbiotic RNe within a few days should allow us to assess the possibility of high electron density, with all its implications for the emission site, emission measure, and the inferred clumpiness in the outflow. Perhaps in V3890 Sgr a first mechanism of shock production in a small volume close to the red giant evolved into shocks occurring farther from the red giant at a later epoch, and in a medium of much lower density, as inferred in the observations of V745 Sco and RS Oph, and this may also explain the longer-than-predicted cooling time estimated with the Swift XRT. Further research is also encouraged in modeling our existing data, e.g., with a detailed hydrodynamical calculations, to seek the most appropriate physical model. 
High-resolution X-ray spectroscopy of nova ejecta has a rich potential to lead to discoveries in nova physics. We suggest these observations should be made whenever this is possible to widen the database and extend it also to nonsymbiotic and classical novae. We foresee that the Jaxa, NASA, and ESA X-Ray Imaging and Spectroscopic Mission XRISM (see Williams et al. 2019) will produce results with even higher S/N, and later Athena (see D'Andrea 2019) will allow much shorter exposure times, thereby extending this type of work to many more novae at repeated epochs, to make comparison with outburst properties at other wavelengths and construct a coherent picture of the challenging puzzle of the nova physics.

We thank Dina Prialnik for sharing unpublished results with us. M.O. and J.D. acknowledge support of a Chandra award to work on this observation, obtained as Director Discretionary Target of Opportunity. We are grateful to Belinda Wilkes, Chandra X-Ray center director, for scheduling this observation on short notice. Other team members acknowledge support from the following funding agencies: E.B. by a Center of Excellence of THE ISRAEL SCIENCE FOUNDATION (grant No. 2752/19); G.J.M.L as a member of the CIC-CONICET (Argentina), from grant PICT 0901/207; J.M. from the National Science Centre, Poland, through grant OPUS 2017/ 27/B/ST9/01940; N.P.M.K. and K.L.P. from the UK Space Agency; S.S. from NASA support to ASU; M.J.D. from the UK Science and Technology Facilities Council (STFC); R.D. G. from NASA and the United States Air Force (USAF); and C.E.W. from NASA.

\section{ORCID iDs}

M. Orio (i) https://orcid.org/0000-0003-1563-9803

J. J. Drake (i) https://orcid.org/0000-0002-0210-2276

J.-U. Ness (iD https://orcid.org/0000-0003-0440-7193

E. Behar (10) https://orcid.org/0000-0002-9356-1645

G. J. M. Luna (iD https://orcid.org/0000-0002-2647-4373

M. J. Darnley (iD https://orcid.org/0000-0003-0156-3377

J. Gallagher (ib https://orcid.org/0000-0001-8608-0408

R. D. Gehrz (i) https://orcid.org/0000-0003-1319-4089

N. P. M. Kuin (i) https://orcid.org/0000-0003-4650-4186

J. Mikolajewska (1) https://orcid.org/0000-0003-3457-0020

N. Ospina (i) https://orcid.org/0000-0002-8404-1808

K. L. Page (i) https://orcid.org/0000-0001-5624-2613

R. Poggiani (i) https://orcid.org/0000-0002-9968-2464

S. Starrfield (i) https://orcid.org/0000-0002-1359-6312

R. Williams (1) https://orcid.org/0000-0002-3742-8460

C. E. Woodward (iD https://orcid.org/0000-0001-6567-627X

\section{References}

Abdo, A. A., Ackermann, M., Ajello, M., et al. 2010, Sci, 329, 817 Anupama, G. C., \& Mikołajewska, J. 1999, A\&A, 344, 177

Asplund, M., Grevesse, N., Sauval, A. J., \& Scott, P. 2009, ARA\&A, 47, 481 Banerjee, D. P. K., Joshi, V., Venkataraman, V., et al. 2014, ApJL, 785, L11 Bautista, M. A., \& Kallman, T. R. 2000, ApJ, 544, 581

Belczynski, K., \& Mikolajewska, J. 1998, MNRAS, 296, 77

Bode, M. F. 1987, in RS Ophiuchi (1985) and the Recurrent Nova Phenomenon, ed. M. F. Bode (Utrecht: VNU Science Press), 241

Bode, M. F., Darnley, M. J., Beardmore, A. P., et al. 2016, ApJ, 818, 145

Bondi, H., \& Hoyle, F. 1944, MNRAS, 104, 273

Brandi, E., Quiroga, C., Mikołajewska, J., Ferrer, O. E., \& García, L. G. 2009, A\&A, 497, 815

Buil, C. 2006, CBET, 403, 1

Buson, S., Jean, P., \& Cheung, C. C. 2019, ATel, 13114, 1

Cheung, C. C., Jean, P., \& Shore, S. N. 2014, ATel, 5879, 1
D'Andrea, M. 2019, arXiv:1904.03307

del Zanna, G., Dere, K. P., Young, P. R., Landi, E., \& Mason, H. E. 2015 A\&A, 582, A56

della Valle, M., \& Livio, M. 1995, ApJ, 452, 704

Dorman, B., \& Arnaud, K. A. 2001, in ASP Conf. Ser. 238, Redesign and Reimplementation of XSPEC, ed. J. Harnden, F. A. Primini, \& H. E. Payne (San Francisco, CA: ASP), 415

Drake, J. J., Delgado, L., Laming, J. M., et al. 2016, ApJ, 825, 95

Drake, J. J., Laming, J. M., Ness, J. U., et al. 2009, ApJ, 691, 418

Evans, A., Banerjee, D. P. K., Geballe, T. R., et al. 2019, ATel, 13088, 1

Fruscione, A., McDowell, J. C., Allen, G. E., et al. 2006, Proc. SPIE, 6270 $62701 \mathrm{~V}$

Gabriel, A. H., \& Jordan, C. 1969, MNRAS, 145, 241

Gehrz, R. D., \& Ney, E. P. 1990, PNAS, 87, 4354

Harrison, T. E., Johnson, J. J., \& Spyromilio, J. 1993, AJ, 105, 320

Hillman, Y., Prialnik, D., Kovetz, A., Shara, M. M., \& Neill, J. D. 2014, MNRAS, 437, 1962

Iłkiewicz, K., Mikołajewska, J., Miszalski, B., et al. 2019, A\&A, 624, A133

Iłkiewicz, K., Mikołajewska, J., Stoyanov, K., Manousakis, A., \& Miszalski, B. 2016, MNRAS, 462, 2695

José, J., \& Hernanz, M. 1998, ApJ, 494, 680

Kelly, K. J., Iliadis, C., Downen, L., José, J., \& Champagne, A. 2013, ApJ, 777, 130

Kuin, P., Darnley, M., Drake, J., et al. 2019, ATel, 13072, 1

Lines, H. C., Lines, R. D., \& McFaul, T. G. 1988, AJ, 95, 1505

Luna, G. J. M., Mukai, K., Sokoloski, J. L., et al. 2018, A\&A, 619, A61

Luna, G. J. M., Pillitteri, I., Mukai, K., \& Sokoloski, J. L. 2014, ATel, 5881, 1

Luna, G. J. M., Sokoloski, J. L., \& Mukai, K. 2008, in ASP Conf. Ser. 401, High Energy X-ray Emission from Recurrent Novae in Quiescence: T CrB, ed. A. Evans et al. (San Francisco, CA: ASP), 342

Luna, G. J. M., Sokoloski, J. L., Mukai, K., \& Nelson, T. 2013, A\&A, 559, A6

Metzger, B. D., Finzell, T., Vurm, I., et al. 2015, MNRAS, 450, 2739

Mikołajewska, J. 2012, BaltA, 21, 5

Mikołajewska, J., \& Shara, M. M. 2017, ApJ, 847, 99

Mohamed, S., Booth, R., \& Podsiadlowski, P. 2013, in IAU Symp. 281, Binary Paths to Type Ia Supernovae Explosions, ed. R. Di Stefano, M. Orio, \& M. Moe (Cambridge: Cambridge Univ. Press), 195

Moore, K., \& Bildsten, L. 2012, ApJ, 761, 182

Mróz, P., Poleski, R., Udalski, A., et al. 2014, MNRAS, 443, 784

Mróz, P., Udalski, A., Poleski, R., et al. 2016, ApJS, 222, 9

Mukai, K., Nelson, T. J., Chomiuk, L., et al. 2019, AAS/HEAD Meeting, 17, 403.06

Munari, U., \& Walter, F. M. 2019a, ATel, 13081, 1

Munari, U., \& Walter, F. M. 2019b, ATel, 13099, 1

Munari, U., \& Walter, F. M. 2019c, ATel, 13069, 1

Mürset, U., \& Schmid, H. M. 1999, A\&AS, 137, 473

Neff, J. S., Smith, V. V., \& Ketelsen, D. A. 1978, ApJS, 38, 89

Nelson, T., Mukai, K., Li, K.-L., et al. 2019, ApJ, 872, 86

Nelson, T., Mukai, K., Orio, M., Luna, G. J. M., \& Sokoloski, J. L. 2011, ApJ, 737, 7

Nelson, T., Orio, M., Cassinelli, J. P., et al. 2008, ApJ, 673, 1067

Ness, J. U., Drake, J. J., Starrfield, S., et al. 2009, AJ, 137, 3414

Ness, J. U., Orio, M., Starrfield, S., et al. 2019, ATel, 13124, 1

Ness, J. U., Schwarz, G. J., Retter, A., et al. 2007, ApJ, 663, 505

O’Brien, T. J., Bode, M. F., \& Kahn, F. D. 1992, MNRAS, 255, 683

Orio, M. 1993, A\&A, 274, L41

Orio, M. 2013, AstRv, 8, 71

Orio, M. 2015, in The Golden Age of Cataclysmic Variables and Related Objects - III (Golden2015) (Trieste: SISSA), 64

Orio, M., Covington, J., \& Ögelman, H. 2001, A\&A, 373, 542

Orio, M., Rana, V., Page, K. L., Sokoloski, J., \& Harrison, F. 2015, MNRAS, 448, L35

Orio, M., Zezas, A., Munari, U., Siviero, A., \& Tepedelenlioglu, E. 2007, ApJ, 661,1105

Orlando, S., \& Drake, J. J. 2012, MNRAS, 419, 2329

Orlando, S., Drake, J. J., \& Laming, J. M. 2009, A\&A, 493, 1049

Orlando, S., Drake, J. J., \& Miceli, M. 2017, MNRAS, 464, 5003

Osborne, J. P., Page, K. L., Beardmore, A. P., et al. 2011, ApJ, 727, 124

Page, K. L., Beardmore, A. P., Osborne, J. P., et al. 2019, ATel, 13084, 1

Page, K. L., Osborne, J. P., Kuin, N. P. M., et al. 2015, MNRAS, 454, 3108

Pavana, M., Anupama, G. C., \& Kumar, S. P. 2019a, ATel, 13245, 1

Pavana, M., Roy, N., Anupama, G. C., Singh, K. P., \& Girish, V. 2019b, ATel, 13092, 1

Peretz, U., Orio, M., Behar, E., et al. 2016, ApJ, 829, 2

Polisensky, E., Linford, J. D., Giacintucci, S., et al. 2019, ATel, 13185, 1

Porquet, D., Dubau, J., \& Grosso, N. 2010, SSRv, 157, 103 
Prialnik, D. 1986, ApJ, 310, 222

Rudy, R., Subasavage, J., Crawford, K., et al. 2019, ATel, 13059, 1 Schaefer, B. E. 2010, ApJS, 187, 275

Shafter, A. W., Henze, M., Rector, T. A., et al. 2015, ApJS, 216, 34

Shara, M. M., Prialnik, D., Hillman, Y., \& Kovetz, A. 2018, ApJ, 860, 110

Smith, R. K., Brickhouse, N. S., Liedahl, D. A., \& Raymond, J. C. 2001, ApJL, 556, L91

Sokoloski, J. L., Luna, G. J. M., Mukai, K., \& Kenyon, S. J. 2006, Natur, 442, 276

Sokolovsky, K. V., Orio, M., Page, K. L., et al. 2019, ATel, 13050, 1

Stanishev, V., Zamanov, R., Tomov, N., \& Marziani, P. 2004, A\&A, 415, 609

Starrfield, S., Bose, M., Iliadis, C., et al. 2019, arXiv:1910.00575
Starrfield, S., Iliadis, C., Hix, W. R., Timmes, F. X., \& Sparks, W. M. 2009, ApJ, 692, 1532

Strader, J., Chomiuk, L., Aydi, E., et al. 2019, ATel, 13047, 1

Strope, R. J., Schaefer, B. E., \& Henden, A. A. 2010, AJ, 140, 34

Tofflemire, B. M., Orio, M., Page, K. L., et al. 2013, ApJ, 779, 22

Vurm, I., \& Metzger, B. D. 2018, ApJ, 852, 62

Williams, B. J., Kelley, R., \& Petre, R. 2019, AAS/HEAD Meeting, 17, 303.01

Wilms, J., Allen, A., \& McCray, R. 2000, ApJ, 542, 914

Woodward, C. E., Banerjee, D. P. K., Evans, A., Geballe, T. R., \& Starrfield, S. 2019, ATel, 13096, 1

Yaron, O., Prialnik, D., Shara, M. M., \& Kovetz, A. 2005, ApJ, 623, 398

Orio, M., Behar, E., \& Gallagher, J. 2013, MNRAS, 429, 1342 